\title{
LKB1 loss links serine metabolism to DNA methylation and tumorigenesis
}

Filippos Kottakis ${ }^{1,2,3}$, Brandon N. Nicolay ${ }^{1,3}$, Ahlima Roumane ${ }^{1,2,3}$, Rahul Karnik ${ }^{4,5,6}$, Hongcang Gu 4,5,6 , Julia M. Nagle , $^{1,2,3}$, Myriam Boukhali, 1,3 , Michele C. Hayward ${ }^{7}$, Yvonne Y. Li ${ }^{8,9}$, Ting Chen ${ }^{8,9,10}$, Marc Liesa ${ }^{11,12}$, Peter S. Hammerman ${ }^{8,9,13}$, Kwok Kin Wong ${ }^{8,9,10}$, D. Neil Hayes ${ }^{7}$, Orian S. Shirihai ${ }^{11,12}$, Nicholas J. Dyson ${ }^{1,3}$, Wilhelm Haas ${ }^{1,3}$, Alexander Meissner ${ }^{4,5,6}$ \& Nabeel Bardeesy ${ }^{1,2,3}$

Intermediary metabolism generates substrates for chromatin modification, enabling the potential coupling of metabolic and epigenetic states. Here we identify a network linking metabolic and epigenetic alterations that is central to oncogenic transformation downstream of the liver kinase B1 (LKB1, also known as STK11) tumour suppressor, an integrator of nutrient availability, metabolism and growth. By developing genetically engineered mouse models and primary pancreatic epithelial cells, and employing transcriptional, proteomics, and metabolic analyses, we find that oncogenic cooperation between LKB1 loss and KRAS activation is fuelled by pronounced mTOR-dependent induction of the serine-glycineone-carbon pathway coupled to $S$-adenosylmethionine generation. At the same time, DNA methyltransferases are upregulated, leading to elevation in DNA methylation with particular enrichment at retrotransposon elements associated with their transcriptional silencing. Correspondingly, LKB1 deficiency sensitizes cells and tumours to inhibition of serine biosynthesis and DNA methylation. Thus, we define a hypermetabolic state that incites changes in the epigenetic landscape to support tumorigenic growth of LKB1-mutant cells, while resulting in potential therapeutic vulnerabilities.

Substrates and inhibitors of chromatin-modifying enzymes are generated in intermediary metabolism, so changes in nutrient availability and utilization can influence epigenetic regulation ${ }^{1,2}$. Importantly, recent studies have indicated that the interplay between metabolism and epigenetics can serve as a programmed switch in cell states. For example, mouse embryonic stem cell differentiation is promoted by succinate-mediated inhibition of histone demethylases (HDMs) and TET DNA demethylases ${ }^{3}$, or by decreased $S$-adenosyl-methionine (SAM) levels leading to loss of histone H3K4 methylation ${ }^{4}$. Moreover, aberrant metabolic activity can produce pathological effects by altering chromatin regulation. Most notably, mutations in the genes encoding the isocitrate dehydrogenase (IDH) 1 and IDH2 enzymes lead to the generation of 2-hydroxyglutarate, which inhibits HDMs and TETs and thereby alters DNA and histone methylation-changes that have been implicated in overriding cell differentiation and promoting tumorigenesis ${ }^{5}$. Whether this paradigm extends more generally to other oncogenic mutations remains unclear, and this question has implications for understanding cancer pathogenesis and developing improved treatments. Here, we demonstrate that dynamic exchange between metabolism and chromatin regulation contributes to pancreatic tumorigenesis driven by mutation of the LKB1 serine-threonine kinase.

LKB1 is mutationally inactivated in a range of sporadic cancers, including pancreatic carcinomas ${ }^{6-8}$. Additionally, germline mutations in LKB1 cause Peutz-Jeghers syndrome, which comprises gastrointestinal polyps and a high incidence of gastrointestinal tract carcinomas (for example, an approximately 100 -fold increase in pancreatic cancer $)^{9,10}$. Cancers with LKB1 mutations tend to exhibit aggressive clinical features and different therapeutic sensitivity from cancers without these mutations ${ }^{11-14}$. LKB1 directly activates a family of 14 kinases related to AMP-activated protein kinase (AMPK), many of which are coupled to nutrient sensing and broadly reprogram cell metabolism ${ }^{15}$. Thus, metabolic rewiring is thought to be a driver of tumorigenesis after LKB1 loss. We now identify an LKB1-regulated program that links metabolic alterations to control of the epigenome and is involved in malignant growth. Our results provide evidence that coupled metabolic and epigenetic states have a more general role in cancer pathogenesis and suggest therapeutic strategies that could target these intersecting processes.

\section{Synergy between LKB1 and KRAS mutations}

LKB1 inactivation frequently coincides with mutations in the RAS-RAF pathway in human cancers and these genetic alterations cooperate to drive tumorigenesis in genetically engineered mouse models $(\text { GEMMs })^{6,11,14,16}$. We examined the interactions between oncogenic KRAS $^{\mathrm{G} 12 \mathrm{D}}$ and deletion of LKB1 in adult pancreatic ducts using a tamoxifen-inducible GEMM (Extended Data Fig. 1a). The combined alterations resulted in pancreatic cancers by $20-25$ weeks, whereas the individual mutations had no pathological effects at this age (Fig. 1a and Extended Data Fig. 1b). To investigate the mechanisms of tumorigenesis, we isolated primary pancreatic ductal epithelial cells from mice with conditional KRAS ${ }^{\mathrm{G} 12 \mathrm{D}}$ and LKB1 alleles ( $n=2$ lines per genotype) and transduced them with adenoviruses expressing Cre and/or Flp recombinase to generate $\mathrm{KRAS}^{\mathrm{G} 12 \mathrm{D} /+}, \mathrm{LKB}^{-/-}$and $\mathrm{KRAS}^{\mathrm{G} 12 \mathrm{D} /+} ; \mathrm{LKB}^{-/-}$ cells (K, L and KL cells, respectively) as well as wild-type parental lines (Extended Data Fig. 1c). Only KL cells were tumorigenic following injection into severe combined immunodeficient (SCID) mice or growth in soft agar, and tumorigenicity was blocked by restoration of

\footnotetext{
${ }^{1}$ Cancer Center, Massachusetts General Hospital, 185 Cambridge Street, Boston, Massachusetts 02114, USA. ${ }^{2}$ Center for Regenerative Medicine, Massachusetts General Hospital, 185 Cambridge Street, Boston, Massachusetts 02114, USA. ${ }^{3}$ Department of Medicine, Harvard Medical School, Boston, Massachusetts 02114, USA. ${ }^{4}$ Broad Institute of MIT and Harvard, Cambridge, Massachusetts 02142, USA. ${ }^{5}$ Harvard Stem Cell Institute, Cambridge, Massachusetts 02138, USA. ${ }^{6}$ Department of Stem Cell and Regenerative Biology, Harvard University, Cambridge, Massachusetts 02138, USA. 7 UNC, Lineberger Comprehensive Cancer Center, Chapel Hill, North Carolina 27599, USA. ${ }^{8}$ Department of Medicine, Brigham and Women's Hospital and Harvard Medical School, Boston, Massachusetts 02115, USA. ${ }^{9}$ Department of Medical Oncology, Dana Farber Cancer Institute, Boston, Massachusetts 02215, USA. ${ }^{10}$ Belfer Institute for Applied Cancer

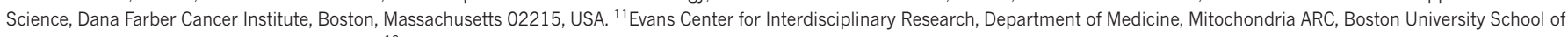
Medicine, Boston, Massachusetts 02118, USA. ${ }^{2}$ Department of Medicine, Division of Endocrinology, Diabetes and Hypertension, UCLA David Geffen School of Medicine, Los Angeles, California 90095, USA. ${ }^{13}$ Cancer Program, Broad Institute of Harvard and MIT, Cambridge, Massachusetts 02142, USA.
} 

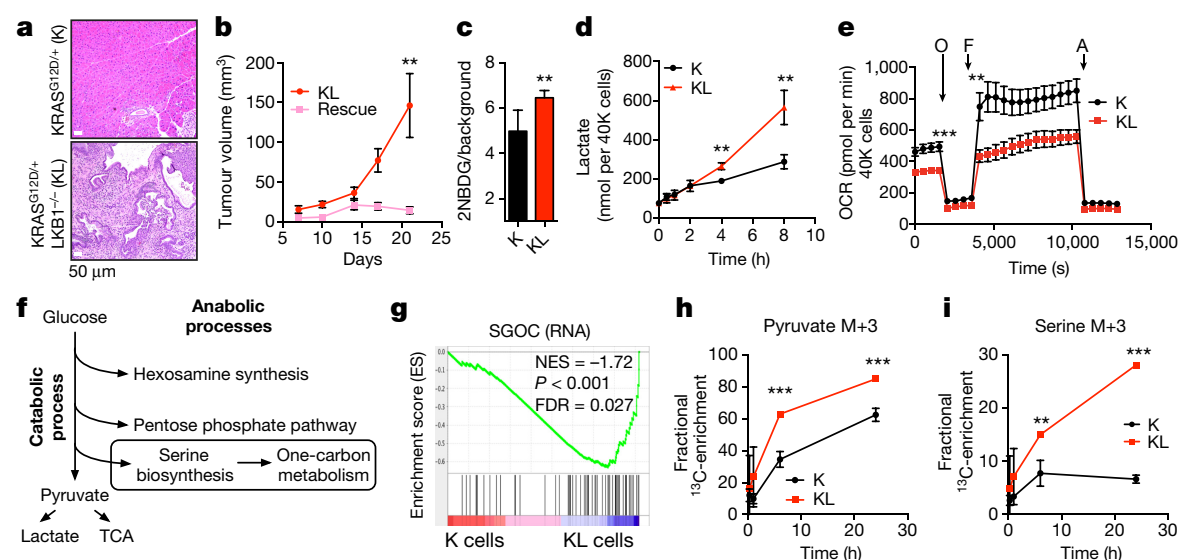

Figure $1 \mid$ LKB1 inactivation synergizes with $\mathrm{KRAS}^{\mathrm{G} 12 \mathrm{D}}$ to potentiate glycolysis, serine metabolism, and tumorigenesis. a, Representative pancreas histology of the indicated genotypes of mice at 20-25 weeks ( $n=4$ per genotype). b, Subcutaneous tumour growth of KL cells expressing empty vector (KL) or LKB1 (rescue) ( $n=8$ per group). c, d, Ductal cells tested for glucose uptake (c; $n=6$, independent replicates), and lactate secretion (d; $n=3)$. e, Oxygen consumption rates

wild-type LKB1 (Fig. $1 \mathrm{~b}$ and Extended Data Fig. 1d-g). In vitro, KL cells showed greater proliferation than K cells, and both showed greater proliferation than wild-type or L cells (Extended Data Fig. $1 \mathrm{~h}-\mathrm{j}$ ). Thus, primary ductal cells provide a tractable in vitro system to study mechanisms of epithelial cell transformation arising from LKB1 inactivation.

Focusing on the metabolic alterations provoked by loss of LKB1, we found that KL cells exhibited an approximately $30 \%$ increase in glucose uptake compared to K cells, and showed marked elevations in levels of the GLUT1 transporter and ATP (Fig. 1c and Extended Data Fig. 1k, 1). Lactate levels were elevated in KL cells, whereas oxygen consumption and citrate levels were reduced (Fig. 1d, e and Extended Data Fig. 1m). Moreover, KL cells showed heightened sensitivity to acute glucose deprivation and to inhibition of glycolysis using the glucose analogue 2-deoxyglucose, the pyruvate dehydrogenase kinase inhibitor dichloroacetate, or the lactate dehydrogenase inhibitor galloflavin (Extended Data Fig. $1 \mathrm{n}-\mathrm{q})$. Importantly, neither KRAS ${ }^{\mathrm{G} 12 \mathrm{D}}$ nor LKB1 inactivation alone promoted significant alterations in glucose metabolism in $\mathrm{K}$ and KL cells under nutrient-replete conditions $(n=21)$. $\mathbf{f}$, Fates of glycolytic intermediates. $g$, GSEA showing enrichment of serine-glycineone-carbon network ${ }^{18}$ (K cells, $n=3$; KL cells, $n=4$ ). NES, normalized enrichment score. $\mathbf{h}, \mathbf{i}$, Isotopomer abundance of $\mathrm{U}\left[{ }^{13} \mathrm{C}\right]$ glucose-derived $\mathrm{M}+3$ pyruvate $(\mathbf{h})$ or serine ( $\mathbf{i} ; n=3$, biological replicates). Data pooled from three (e) or representative of two (d) experiments. Error bars, s.e.m. (b), s.d. (c, d, g, h). $* P<0.05, * * P<0.01$, *** $P<0.001$.

(Extended Data Fig. 1r-t). Thus, these genetic lesions acted synergistically to potentiate glycolysis while rendering cells highly dependent on glucose availability.

These data suggested that an increased supply of glycolytic intermediates was available for anabolic processes to support the growth of KL cells (Fig. 1f). Notably, gene set enrichment analysis (GSEA) of RNA-sequencing (RNA-seq) and quantitative proteomics ${ }^{17}$ data indicated that KL cells are enriched for glycolytic enzymes and for networks that connect glycolytic intermediates to one-carbon metabolism, with serine-glycine-threonine and folate metabolism scoring highly among the induced pathways (Supplementary Data Table 1). There was particularly striking enrichment of a 64-gene signature defining the entire serine-glycine-one carbon (SGOC) network ${ }^{18}$, indicating strong coordinate activation of these pathways (Fig. 1g and Extended Data Fig. 2a). Accordingly, the use of uniformly carbon-13-labelled glucose $\left(\mathrm{U}\left[{ }^{13} \mathrm{C}\right]\right.$ glucose $)$ demonstrated that KL cells show augmented production of glucose-derived pyruvate and lactate and an even more

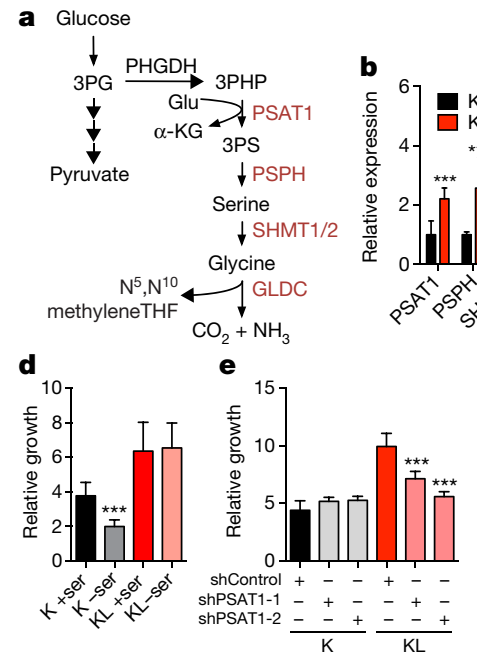

Figure 2 | Activation of de novo serine biosynthesis supports growth of LKB1-deficient cells. a, Serine biosynthesis pathway. Red, upregulated in KL cells. b, Serine pathway gene expression ( $n=8$ per genotype). c, Isotopomer abundance of $\left[{ }^{15} \mathrm{~N}\right]$ glutamine-derived $\mathrm{M}+1$ serine and glycine ( $n=3$, biological replicates). d, e, Three-day growth of ductal cells cultured with or without $0.4 \mathrm{mM}$ serine $(\mathbf{d} ; n=20)$ or transduced with the indicated shRNAs $(\mathbf{e} ; n=6)$. f, Six-day proliferation of KL cells
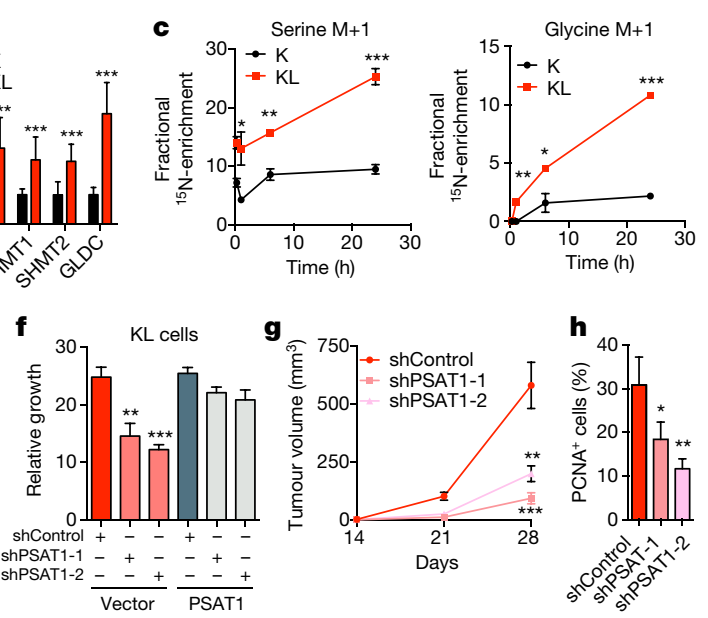

transduced with the indicated shRNAs and expression constructs $(n=3)$. g, Subcutaneous growth of tumours from KL cells transduced with the indicated shRNAs ( $n=12$ tumours per group). $\mathbf{h}$, Proportion of $\mathrm{CK} 19^{+}$ tumours cells that are $\mathrm{PCNA}^{+}(\operatorname{shControl} n=4, \operatorname{shPSAT} 1-1 n=4$, shPSAT1-2 $n=3$, representative tumours). Data pooled from four (b) or representative of two (e, f) or four (d) experiments. Error bars: s.d. (b-f), s.e.m. (g, h). $* P<0.05, * * P<0.01, * * * P<0.001$. 
a

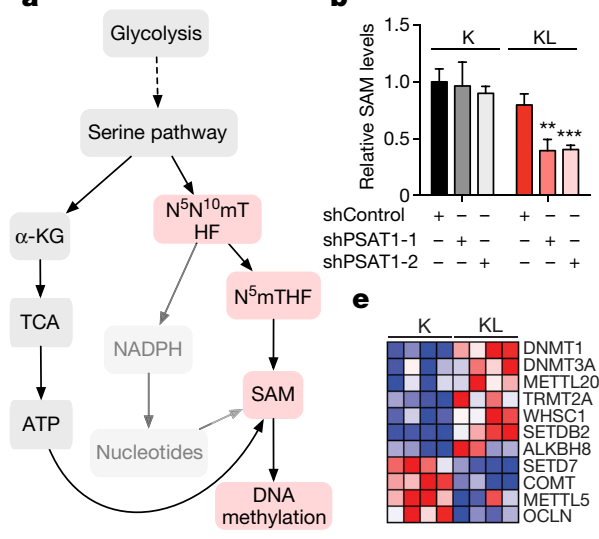

\section{c}
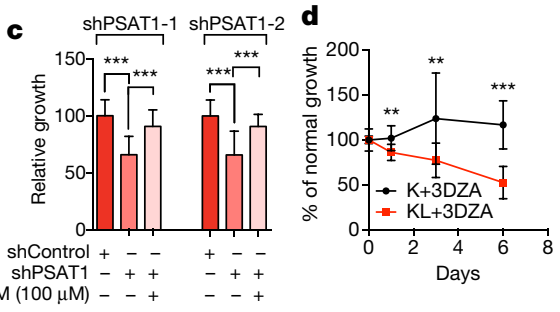

f $K$ cells
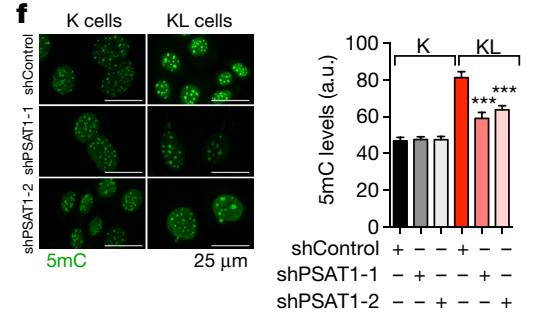

(3-DZA) ( $n=12)$. e, Heatmap of differentially regulated SAM-using enzymes (proteomics). f, $5 \mathrm{mC}$ in ductal cells ( $77-177$ cells). Data pooled from two $(\mathbf{c}, \mathbf{d}, \mathbf{f})$ or representative of two $(\mathbf{b})$ experiments. Error bars: s.d. (b-d), s.e.m. (f). $* P<0.05, * * P<0.01, * * * P<0.001$.

Similar results were obtained after treatment with aminooxyacetate (AOA), an inhibitor of PSAT1 and other aminotransferases (Extended Data Fig. 4e, f). Thus, the requirement of KL cells for PSAT1 appears to be independent of its roles in redox homeostasis or maintenance of nucleoside pools.

Serine metabolism can also fuel the methionine salvage pathway (Fig. 3a), which is the main mechanism of production for the methyl donor $S$-adenosyl methionine (SAM) $)^{21,22}$. Notably, multiple enzymes that channel serine metabolism intermediates into the methionine salvage pathway and that contribute to SAM biosynthesis were upregulated in KL cells (Extended Fig. 4g). Accordingly, PSAT1 knockdown reduced SAM levels in KL cells but not in K cells (Fig. 3b). Furthermore, SAM supplementation mitigated the proliferation defects caused by PSAT1 ablation in KL cells (Fig. 3c). Conversely, suppression of SAM biosynthesis by inhibition of $S$-adenosyl-homocysteine-hydrolase (SAH) with 3-deazaadenosine (3DZA), or of methionine-adenosyltransferase (MAT) with cycloleucine, slowed the growth of KL cells, whereas K cells remained unaffected (Fig. 3d and Extended Data Fig. 4h). Thus, activation of de novo serine biosynthesis contributes to SAM production to support growth of LKB1-deficient cells, possibly by providing active methyl groups in the form of $\mathrm{N}^{5}$-methyl-THF, or by promoting ATP generation (for example, via PSAT1-dependent TCA cycle anaplerosis) $)^{22,23}$.

SAM is the substrate for methylation of lipids, DNA, RNA, metabolites and proteins. Examination of our RNA-seq and proteomics data sets for the expression of the 183 annotated SAM-dependent methyltransferase enzymes revealed significant upregulation of the DNA methyltransferases DNMT1 and DNMT3A in KL cells, whereas few of the other enzymes showed any changes (Fig. 3e, Extended Data Fig. 5a and Supplementary Data Table 2). Quantitative PCR and immunoblot analyses verified the regulation of expression of these enzymes by LKB1 (Extended Data Fig. 5b, c). Importantly, inhibition of DNA methylation using 5-aza-2-deoxycytidine (decitabine) or the nonnucleoside DNMT inhibitor RG108 promoted SAM accumulation in KL cells but not in K cells (Extended Data Fig. 5d), suggesting that DNMTs are major SAM-utilizing enzymes in the context of LKB1 deficiency.

Accordingly, immunofluorescence and DNA dot blot analysis demonstrated that KL cells had a significant increase in 5-methylcytosine $(5 \mathrm{mC})$ as compared to $\mathrm{K}$ cells, an effect that was reversed by LKB1 rescue (Extended Data Fig. 5e-g). By contrast, there were no consistent changes in global levels of a series of histone methylation marks, in 5-hydroxy-methylcytosine, or in $\mathrm{N}_{6}$-methyladenosine (Extended Data Fig. 5h-i and data not shown). Notably, PSAT1 knockdown suppressed 

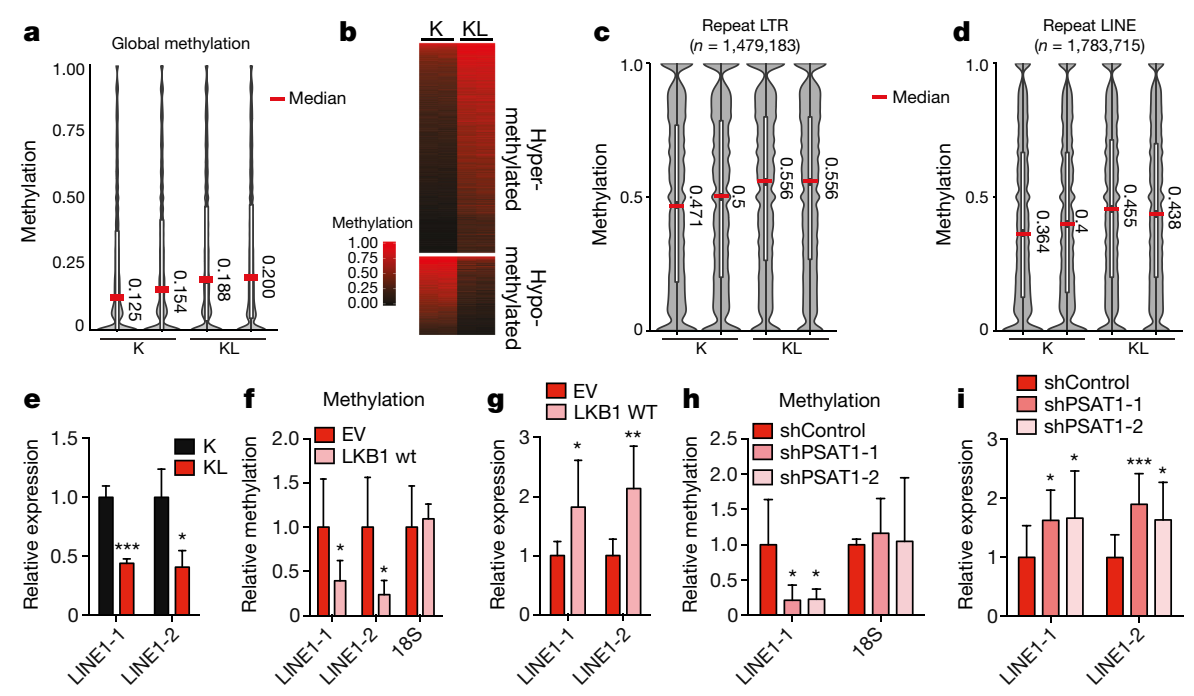

Figure 4 | The LKB1-PSAT1 pathway controls methylation and expression of retrotransposons. a, Distribution of methylation levels in ductal cells. Median values are indicated. b. Heatmap of differentially methylated tiles. c, d, Distribution of methylation levels in LINE1 (c) and LTR (d) repeats. e, Expression of LINE1 in ductal cells, tested using two independent primer sets $(n=3)$. $\mathbf{f}, \mathbf{g}$, KL cells transduced with EV or LKB1

DNA methylation in KL cells, and this effect was reversed by shRNAresistant PSAT1 cDNA, while K and KPC controls were unaffected. (Fig. $3 \mathrm{f}$ and Extended Data Fig. 6a-c). Comparison of all four genotypes using our set of isogenic ductal cells confirmed that serine pathway activity and DNA methylation were specifically potentiated upon dual KRAS ${ }^{\text {G12D }}$ expression and LKB1 loss (Extended Data Fig. 6d-g).

The expression of a kinase-dead (KD) LKB1 mutant confirmed that the activation of key transcriptional and metabolic circuits in KL cells was due to loss of LKB1 kinase activity (Extended Data Fig. 7a-e). Accordingly, KL cells showed reduced activity of AMPK, an important target of the LKB1 kinase, and consequent activation of mechanistic target of rapamycin complex I (mTORC1) (Extended Data Fig. 7f, w). Moreover, in K cells, AMPK suppression using the small molecule inhibitor dorsomorphin (compound C) or shRNAs targeting AMPK $\alpha 1$ or AMPK $\alpha 2$ increased each of the metabolic and gene expression parameters and upregulated global DNA methylation levels, mimicking the effects of LKB1 loss (Extended Data Fig. 7g-q). Additionally, AMPK silencing blocked the effects of LKB1 re-expression on the growth and metabolic properties of KL cells (Extended Data Fig. 7r-u). Finally, KL cells were hypersensitive to mTOR inhibition using Torin 1, which suppressed the metabolic and transcriptional changes seen in these cells (Extended Data Fig. 7v-z). Thus, deregulation of AMPKmTORC1 signalling contributes to the altered metabolic and epigenetic network in KL cells.

\section{LKB1 loss silences retrotransposons}

DNA methylation can contribute to transcriptional regulation and maintenance of genomic stability ${ }^{24}$. We mapped methylation changes by conducting whole-genome bisulphite sequencing (WGBS) of K and KL cells (two independently derived lines per genotype). The data demonstrated a marked increase in mean CpG methylation in KL cells (Fig. 4a and Extended Data Fig. 8a). Comparison of methylation levels at non-repetitive 100-bp tiles revealed 3,395 hypermethylated and 1,270 hypomethylated regions in KL cells (FDR $<0.05$, methylation difference $>0.1$; Fig. $4 \mathrm{~b}$ and Extended Data Fig. 8b). However, the genes associated with differentially methylated regions had only modest overlap with those showing differential expression, suggesting that methylation of these elements may not prominently affect transcriptional regulation (Extended Data Fig. 8c). Thus, we focused on the repetitive portion of the genome. Notably, methylation in KL cells was particularly enriched at retrotransposon repeats (long terminal assessed for methylation of LINE1 and 18S (f; $n=6$, LINE1-1 and 18S; $n=4$, LINE1-2), and LINE1 expression $(\mathbf{g} ; n=6)$. $\mathbf{h}, \mathbf{i}, \mathrm{KL}$ cells transduced with the indicated shRNAs were assessed for LINE1 and 18S methylation $(\mathbf{h} ; n=6)$ and LINE1 expression $(\mathbf{i} ; n=10)$. Data pooled from two $(\mathbf{f}, \mathbf{h})$ or representative of two $(\mathbf{e}, \mathbf{g}, \mathbf{i})$ experiments. Error bars: s.d. $* P<0.05$, $* * P<0.01, * * * P<0.001$

repeats (LTRs), long interspersed nuclear elements (LINEs) and short interspersed nuclear elements (SINEs)) compared to non-repeat elements (promoters, CpG islands and shores) (Fig. 4c, d and Extended Data Fig. 8d-k). These elements comprise a large proportion of the genome, have promoters that can be actively transcribed when unmethylated, and can influence regulation of host genes ${ }^{25}$. Consistent with a functional role for the observed retrotransposon methylation, KL cells had a $>50 \%$ reduction in LINE-1 expression compared to K cells (Fig. 4e). Moreover, restoration of LKB1 suppressed LINE-1 methylation, as determined by $5 \mathrm{mC}-\mathrm{DNA}$ immunoprecipitation-PCR analysis (meDIP-qPCR), and led to an approximately twofold upregulation of LINE-1 transcript expression (Fig. 4f, g). Importantly, PSAT1 knockdown also reduced LINE-1 methylation and induced its expression in KL cells (Fig. 4h, i), indicating that increased serine metabolism supports retrotransposon methylation and transcriptional silencing downstream of LKB1 loss.

Because retrotransposons can function as important modulators of host gene expression ${ }^{25}$, we explored the correlation between differentially expressed genes in K versus KL cells and the presence of linked retrotransposons. Retrotransposon elements were significantly enriched ( $\chi^{2}$ test, $P<0.0001$ ) in the gene bodies of differentially expressed genes as compared to the distribution of these elements across all genes (Extended Data Fig. 81, m). By contrast, minimal enrichment was observed when promoter regions were considered. Such specificity is notable in light of evidence that intragenic methylation (including repeat methylation) can modify the activity of linked promoters and affect RNA processing ${ }^{25,26}$. Thus, the LKB1-SGOC pathway alters the epigenetic landscape and dynamically regulates retrotransposon methylation and transcriptional activity, changes that appear to be associated with differences in host gene transcription.

\section{KL cells are sensitive to DNMT inhibition}

Consistent with the functional relevance of increased DNA methylation, KL cells were sensitive to shRNA-mediated knockdown of DNMT1 or DNMT3A, whereas K or KPC cells remained largely unaffected (Extended Data Fig. 9a-c). Similar specific sensitivity of KL cells was observed in vivo using doxcycline (dox)-inducible shRNAs to acutely deplete DNMT1 or DNMT3 (or shGFP control) once subcutaneous tumours reached $50 \mathrm{~mm}^{3}$ in size (Fig. $5 \mathrm{a}-\mathrm{c}$ and Extended Data Fig. 9d-f). These data indicate that inhibition of DNA methylation is a potential vulnerability of KL cells. Indeed, KL cells were hypersensitive 

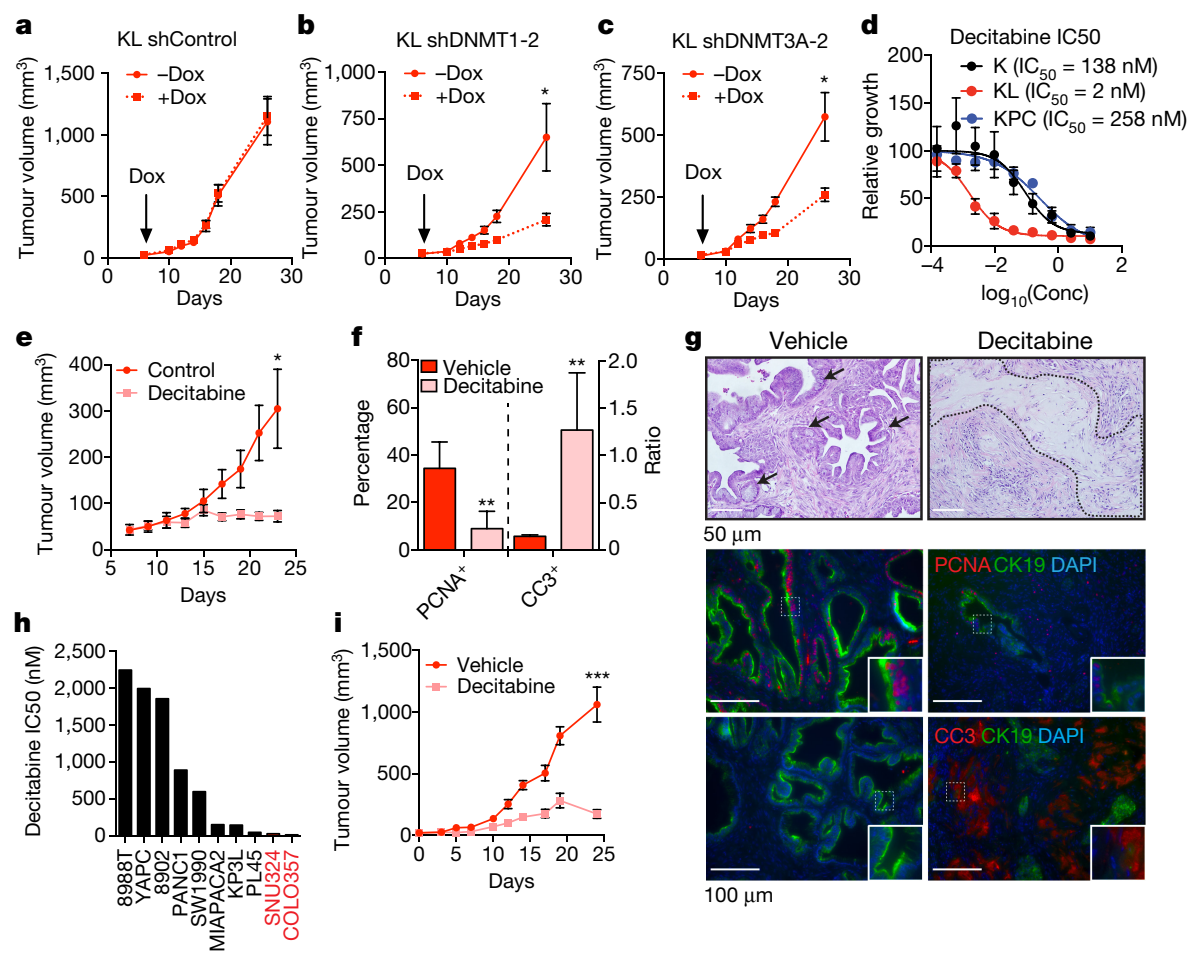

Figure 5 | LKB1 deficiency confers hypersensitivity to DNA methylation inhibitors. a-c, Volume of subcutaneous tumours derived from KL cells transduced with Dox-inducible shRNAs ( $n=6$ tumours per condition). d, Decitabine $\mathrm{IC}_{50}$ for K, KL or KPC cells $(n=4)$. e-g, Decitabine treatment of KL xenografts ( $n=20$ tumours per condition). e, Tumour growth. f, Proportion of $\mathrm{CK}_{1} 9^{+}$tumour cells that are $\mathrm{PCNA}^{+}$ ( $n=4$ representative tumours), and ratio of cleaved-caspase 3 (CC3) to DAPI staining ( $n=4$ representative tumours). g, Haematoxylin and eosin staining of representative tumours (top; arrows: tumour glands, dotted line: hyalinization), and staining for the indicated markers (quantified in $\mathbf{f}$ ). Insets: threefold magnification. $\mathbf{h}$, Decitabine $\mathrm{IC}_{50}$ in human pancreatic cancer cell lines (red, LKB1 mutant; black, LKB1 wild type). i, Growth of COLO357 xenografts treated with decitabine $\left(1 \mathrm{mg} \mathrm{kg}^{-1}\right)$ or vehicle ( $n=6$ per group). Data pooled from three (h) or representative of two (d) experiments. Error bars: s.e.m. $(\mathbf{a}-\mathbf{c}, \mathbf{i})$, s.d. $(\mathbf{d}-\mathbf{f}) . * P<0.05, * * P<0.01$, $* * * P<0.001$.

intermediates are channelled towards the SGOC network leading to increased DNA methylation (Extended Data Fig. 10f). Such interplay between metabolic control and epigenetic reprogramming has been proposed as a key mechanism for cancer development in the context of mutations in metabolic enzymes ${ }^{1,2,5}$. The present work provides evidence for a broader role of metabolic and epigenetic crosstalk in cancer pathogenesis, revealing that LKB1 mutant pancreatic cancer cells have a marked dependency on pathways linking glycolysis, serine metabolism and DNA methylation.

It appears probable that the influence of aberrant metabolism on epigenetics contributes to cancer in the context of other oncogenic mutations. For instance, PI3K/AKT signalling controls acetyl-coenzyme A metabolism to support histone acetylation and regulate growthpromoting genes ${ }^{28}$. Numerous oncogenic pathways rewire metabolism, and the circuits that are activated vary widely, with different genetic lesions favouring distinct fates of glucose, glutamine, fatty acids, and other nutrients. Thus, the resulting alterations in the levels of metabolites affecting chromatin regulation (for example, $\mathrm{NAD}^{+} / \mathrm{NADH}, \mathrm{FAD}$, O-linked $\mathrm{N}$-acetylglucosamine, free fatty acids, SAM and acetyl-CoA) may be fundamental to cancer-promotion. Varying conditions in the tumour microenvironment may alter tumour phenotypes via similar processes $^{29,30}$.

The gain in DNA methylation at retrotransposons that we observed in KL cells is notable in light of the emerging view that these abundant repeat elements serve critical roles in host gene regulation ${ }^{25}$. Moreover, there is increasing evidence that reactivation of silenced retrotransposons may underlie the therapeutic benefit of DNMT inhibitors, serving to induce a type I interferon anti-viral defence program ${ }^{31,32}$. The basis for the widespread differences in response to DNMT inhibitors observed in vitro and in the clinic remains unclear. In this regard, our studies in KRAS mutant pancreatic ductal cells suggest that LKB1 status is a 
genetic marker for DNMT inhibitor responsiveness. Because repeat element methylation does not strictly correlate with sensitivity to these treatments ${ }^{33}$, the precise mechanisms of the specific response of KL cells to DNMT inhibitors remain to be defined. In this setting, it will be of interest to determine the relative contributions of IFN pathway activation and of modulation of host gene expression. Together, our data point to novel therapeutic vulnerabilities in the context of LKB1 mutations, suggesting that it might be possible to use agents targeting nodes of this network in defined patient subsets, although additional studies will be needed to broadly establish these associations in human cancers.

Our findings and other recent reports highlight the importance in cancer of the deregulation of serine biosynthesis, which can result from amplification of genes encoding pathway enzymes ${ }^{23,34}$ or their transcriptional activation downstream of oncogenic drivers, including $\mathrm{NRF}^{35}, \mathrm{c}-\mathrm{MYC}^{36-38}$, and $\mathrm{mTOR}^{39}$. Serine metabolism can fuel diverse biosynthetic pathways and contribute to energy production. Correspondingly, its functional role in aberrant growth appears context dependent, relating variously to supporting nucleotide biosynthesis, NADPH production, TCA cycle anaplerosis, and DNA or histone methylation. These findings underscore the emerging interest in developing new approaches to targeting components of the SGOC network as cancer therapeutics.

Online Content Methods, along with any additional Extended Data display items and Source Data, are available in the online version of the paper; references unique to these sections appear only in the online paper.

\section{Received 27 May 2015; accepted 27 September 2016.}

Published online 31 October 2016.

1. Gut, P. \& Verdin, E. The nexus of chromatin regulation and intermediary metabolism. Nature 502, 489-498 (2013).

2. Etchegaray, J.-P. \& Mostoslavsky, R. Interplay between metabolism and epigenetics: a nuclear adaptation to environmental changes. Mol. Cell $\mathbf{6 2}$, 695-711 (2016).

3. Carey, B. W., Finley, L. W. S., Cross, J. R., Allis, C. D. \& Thompson, C. B. Intracellular $\alpha$-ketoglutarate maintains the pluripotency of embryonic stem cells. Nature 518, 413-416 (2015).

4. Shyh-Chang, N. et al. Influence of threonine metabolism on S-adenosylmethionine and histone methylation. Science 339, 222-226 (2013).

5. Losman, J.-A. \& Kaelin, W. G. Jr. What a difference a hydroxyl makes: mutant IDH, (R)-2-hydroxyglutarate, and cancer. Genes Dev. 27, 836-852 (2013).

6. Waddell, N. et al. Whole genomes redefine the mutational landscape of pancreatic cancer. Nature 518, 495-501 (2015).

7. Witkiewicz, A. K. et al. Whole-exome sequencing of pancreatic cancer defines genetic diversity and therapeutic targets. Nat. Commun. 6, 6744 (2015).

8. Su, G. H. et al. Germline and somatic mutations of the STK11/LKB1 Peutz-Jeghers gene in pancreatic and biliary cancers. Am. J. Pathol. 154, 1835-1840 (1999).

9. Giardiello, F. M. et al. Very high risk of cancer in familial Peutz-Jeghers syndrome. Gastroenterology 119, 1447-1453 (2000).

10. Korsse, S. E. et al. Pancreatic cancer risk in Peutz-Jeghers syndrome patients: a large cohort study and implications for surveillance. J. Med. Genet. 50, 59-64 (2013).

11. Ji, H. et al. LKB1 modulates lung cancer differentiation and metastasis. Nature 448, 807-810 (2007)

12. Chen, Z. et al. A murine lung cancer co-clinical trial identifies genetic modifiers of therapeutic response. Nature 483, 613-617 (2012).

13. Wingo, S. N. et al. Somatic LKB1 mutations promote cervical cancer progression. PLoS One 4, e5137 (2009).

14. Liu, W. et al. LKB1/STK11 inactivation leads to expansion of a prometastatic tumor subpopulation in melanoma. Cancer Cell 21, 751-764 (2012).

15. Shackelford, D. B. \& Shaw, R. J. The LKB1-AMPK pathway: metabolism and growth control in tumour suppression. Nat. Rev. Cancer 9, 563-575 (2009).

16. Cancer Genome Atlas Research Network. Comprehensive molecular profiling of lung adenocarcinoma. Nature 511, 543-550 (2014).

17. Ting, L., Rad, R., Gygi, S. P. \& Haas, W. MS3 eliminates ratio distortion in isobaric multiplexed quantitative proteomics. Nat. Methods 8, 937-940 (2011).

18. Mehrmohamadi, M., Liu, X., Shestov, A. A. \& Locasale, J. W. Characterization of the usage of the serine metabolic network in human cancer. Cell Reports $\mathbf{9}$, 1507-1519 (2014).
19. Bardeesy, N. et al. Both p16(Ink4a) and the p19(Arf)-p53 pathway constrain progression of pancreatic adenocarcinoma in the mouse. Proc. Natl Acad. Sci. USA 103, 5947-5952 (2006).

20. Locasale, J. W. Serine, glycine and one-carbon units: cancer metabolism in full circle. Nat. Rev. Cancer 13, 572-583 (2013).

21. Mentch, S. J. et al. Histone methylation dynamics and gene regulation occur through the sensing of one-carbon metabolism. Cell Metab. 22, 861-873 (2015).

22. Maddocks, O. D. K., Labuschagne, C. F., Adams, P. D. \& Vousden, K. H. Serine metabolism supports the methionine cycle and DNA/RNA methylation through de novo ATP synthesis in cancer cells. Mol. Cell 61, 210-221 (2016).

23. Possemato, R. et al. Functional genomics reveal that the serine synthesis pathway is essential in breast cancer. Nature 476, 346-350 (2011).

24. Schübeler, D. Function and information content of DNA methylation. Nature 517, 321-326 (2015).

25. Elbarbary, R. A., Lucas, B. A. \& Maquat, L. E. Retrotransposons as regulators of gene expression. Science http://dx.doi.org/10.1126/science.aac7247 (2016).

26. Kulis, M., Queirós, A. C., Beekman, R. \& Martín-Subero, J. I. Intragenic DNA methylation in transcriptional regulation, normal differentiation and cancer. Biochim. Biophys. Acta 1829, 1161-1174 (2013).

27. Son, J. et al. Glutamine supports pancreatic cancer growth through a KRAS-regulated metabolic pathway. Nature 496, 101-105 (2013).

28. Lee, J. V. et al. Akt-dependent metabolic reprogramming regulates tumor cell histone acetylation. Cell Metab. 20, 306-319 (2014).

29. Shim, E.-H. et al. L-2-Hydroxyglutarate: an epigenetic modifier and putative oncometabolite in renal cancer. Cancer Discov. 4, 1290-1298 (2014).

30. Intlekofer, A. M. et al. Hypoxia induces production of L-2-hydroxyglutarate. Cell Metab. 22, 304-311 (2015).

31. Roulois, D. et al. DNA-demethylating agents target colorectal cancer cells by inducing viral mimicry by endogenous transcripts. Cell 162, 961-973 (2015).

32. Chiappinelli, K. B. et al. Inhibiting DNA methylation causes an interferon response in cancer via dsRNA including endogenous retroviruses. Cell 162 , 974-986 (2015).

33. Treppendahl, M. B., Kristensen, L. S. \& Grønbæk, K. Predicting response to epigenetic therapy. J. Clin. Invest. 124, 47-55 (2014).

34. Locasale, J. W. et al. Phosphoglycerate dehydrogenase diverts glycolytic flux and contributes to oncogenesis. Nat. Genet. 43, 869-874 (2011).

35. DeNicola, G. M. et al. NRF2 regulates serine biosynthesis in non-small cell lung cancer. Nat. Genet. 47, 1475-1481 (2015).

36. Sun, L. et al. cMyc-mediated activation of serine biosynthesis pathway is critical for cancer progression under nutrient deprivation conditions. Cell Res. 25, 429-444 (2015).

37. Ye, J. et al. Serine catabolism regulates mitochondrial redox control during hypoxia. Cancer Discov. 4, 1406-1417 (2014)

38. Zhang, W. C. et al. Glycine decarboxylase activity drives non-small cell lung cancer tumor-initiating cells and tumorigenesis. Cell 148, 259-272 (2012)

39. Ben-Sahra, I., Howell, J. J., Asara, J. M. \& Manning, B. D. Stimulation of de novo pyrimidine synthesis by growth signaling through mTOR and S6K1. Science 339, 1323-1328 (2013).

Supplementary Information is available in the online version of the paper.

Acknowledgements We thank A. Kimmelman, K. Patra, L. J. Etchegaray, and R. Mostoslavsky for comments on the manuscript, and P. Foltopoulou, B. Martinez and Bardeesy laboratory members for advice. N.B. holds the Gallagher Endowed Chair in Gastrointestinal Cancer Research and received support from the Granara-Skerry Trust, the Linda J. Verville Foundation and the Begg Family, and grants from the NIH (P01 CA117969-07, RO1 CA133557-05). F.K. is supported by a Hirshberg Foundation Career Development Award. F.K. and N.B. were supported by NIH grant P50CA1270003 and are members of the Andrew Warshaw Institute.

Author Contributions F.K. and N.B. conceived and designed the study. F.K., A.R. and J.M.N. performed cell-based and mouse experiments. T.C. assisted with mouse experiments. F.K. and B.N.N. performed and interpreted the tracing experiments. M.B and W.H. performed proteomics. F.K. and M.L. performed the OCR measurements. M.C.H. and D.N.H. provided essential samples and data analysis. Y.Y.L. performed computational analysis. H.G. prepared WGBS libraries. R.K. and A.M. analysed and interpreted the WGBS data. P.S.H., K.K.W., O.S.S. and N.J.D. assisted with data interpretation. F.K. and N.B. wrote the manuscript with feedback from all authors.

Author Information Reprints and permissions information is available at www.nature.com/reprints. The authors declare no competing financial interests. Readers are welcome to comment on the online version of the paper. Correspondence and requests for materials should be addressed to N.B. (bardeesy.nabeel@mgh.harvard.edu).

Reviewer Information Nature thanks M. Rehli and the other anonymous reviewer(s) for their contribution to the peer review of this work. 
a
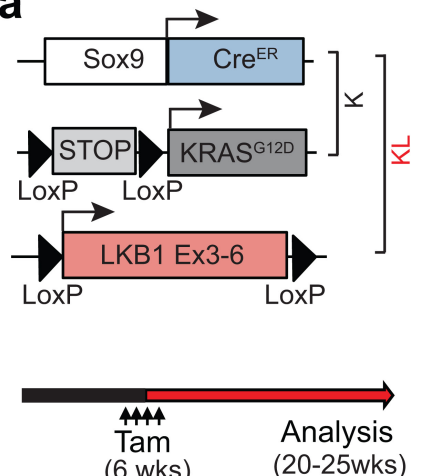

b

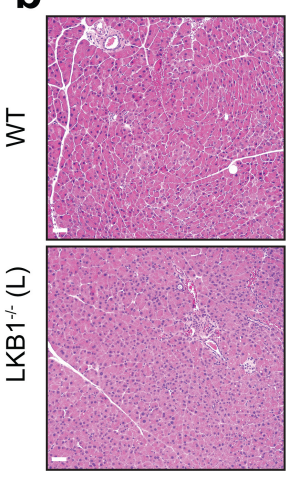

C

FSF/LSL KRAS LKB1 $1^{\text {AL }}$

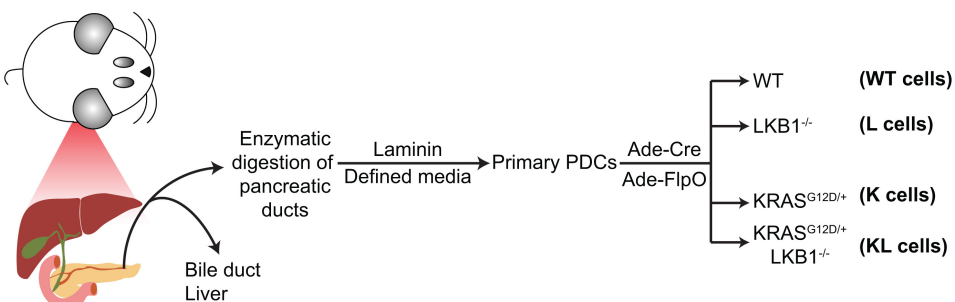

Liver
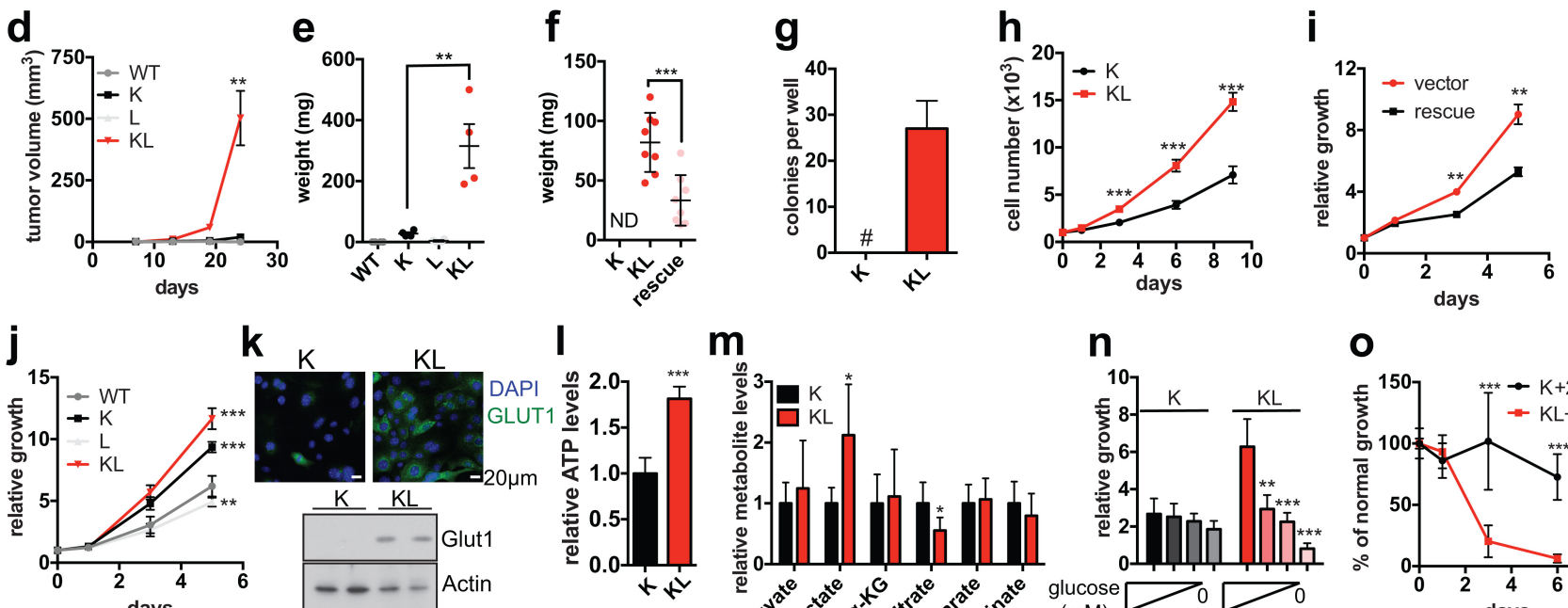

m
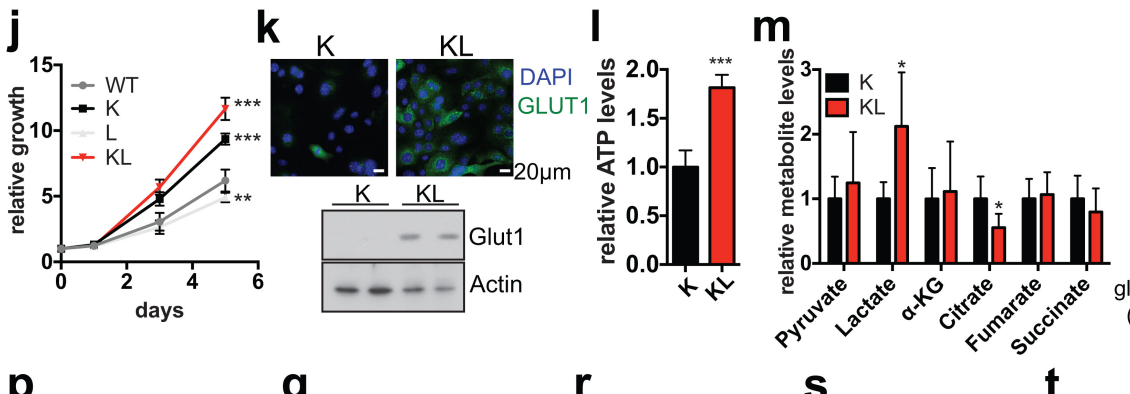

n

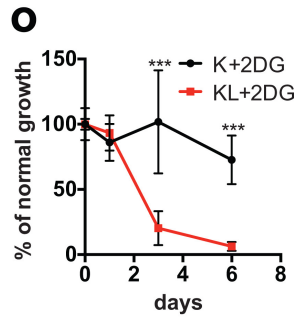

q

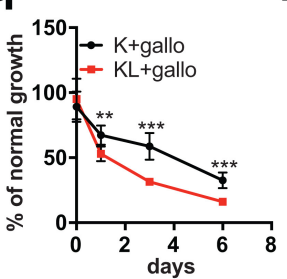

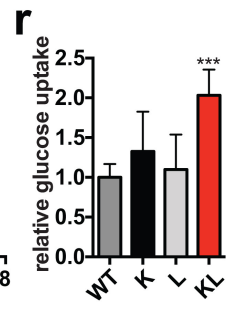

S

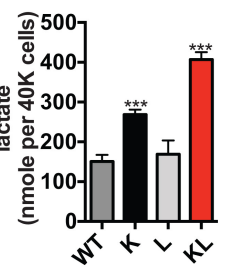

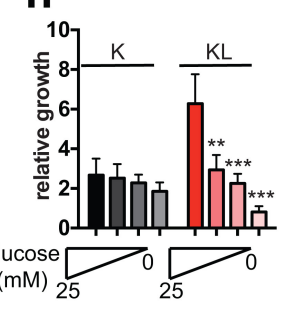

$\mathbf{t}$

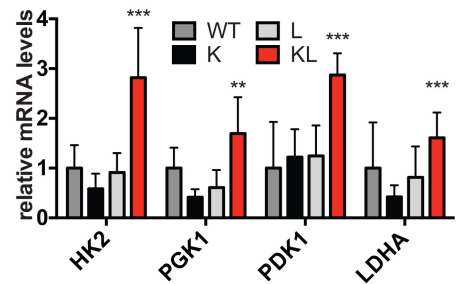

Extended Data Figure 1 | LKB1 suppresses KRAS ${ }^{\text {G12D }}$-driven tumorigenesis and limits glycolysis in primary pancreatic ductal epithelial cells. a, Schematic of GEM models. Sox9-Cre ${ }^{\mathrm{ER}}, \mathrm{LKB} 1^{\mathrm{L} / \mathrm{L}}$, and LSL-KRAS ${ }^{\mathrm{G} 12 \mathrm{D} /+}$ mice were crossed to generate four cohorts: WT $\left(\right.$ Sox9-Cre $\left.{ }^{\mathrm{ER}}\right), \mathrm{L}\left(\right.$ Sox9-Cre $\left.{ }^{\mathrm{ER}} ; \mathrm{LKB1}{ }^{\mathrm{L} / \mathrm{L}}\right), \mathrm{K}\left(\right.$ Sox9-Cre ${ }^{\mathrm{ER}} ; \mathrm{LSL}-\mathrm{KRAS}^{\mathrm{G} 12 \mathrm{D} /+}$ ) and KL (Sox9-Cre $\left.{ }^{\mathrm{ER}} ; \mathrm{LSL}-\mathrm{KRAS}^{\mathrm{G} 12 \mathrm{D} /+} ; \mathrm{LKB1}^{\mathrm{L} / \mathrm{L}}\right)$. Genetic lesions were induced by intraperitoneal injections of tamoxifen at 6 weeks of age, after which mice were observed for signs of disease and killed when $\mathrm{KL}$ animals were moribund (20-25 weeks of age). The WT, K, and L mice had no signs of illness or other abnormalities at this time point. b. Haematoxylin and eosin-stained sections of representative pancreata from WT and L mice ( $n=4$ mice per group). Scale bars, $50 \mu \mathrm{m}$. c, Schematic of primary pancreatic ductal epithelial cell system. Pancreatic ductal epithelial cells were isolated from LSL-KRAS ${ }^{\mathrm{G} 12 \mathrm{D} /+}$ and LSL-KRAS ${ }^{\mathrm{G} 12 \mathrm{D} /{ }^{+}} ; \mathrm{LKB1} 1^{\mathrm{L} / \mathrm{L}}$ mice and infected with Adeno-Cre to generate $\mathrm{K}$ and $\mathrm{KL}$ cells. For studies comparing K, L, KL and WT genotypes, cells were isolated from FSF-KRAS ${ }^{\mathrm{G} 12 \mathrm{D} /{ }^{+}} ; \mathrm{LKB} 1^{\mathrm{L} / \mathrm{L}}$ mice and infected with Adeno-Flipase and/or Adeno-Cre, or neither. d, Volume and $\mathbf{e}$, weight of subcutaneous tumours derived from ductal cells of the indicated genotypes ( $n=4$ tumours per group). Error bars show s.e.m. For source data on tumour volume, see Supplementary Data Table 4. f, Weight of subcutaneous tumours from $\mathrm{K}(n=6), \mathrm{KL}(n=8)$ and $\mathrm{KL}$ cells transduced with retrovirus expressing LKB1 cDNA (rescue, $n=8$ ). g, Number of colonies formed in soft agar by K (none detected) or KL cells ( $n=6$ independent biological replicates). Error bars show s.e.m. h, Proliferation of K and KL cells in nutrient-replete medium $(n=4)$. i, Proliferation of KL cells transduced with retroviruses expressing empty vector or LKB1 (rescue; $n=3$ ). $\mathbf{j}$, Proliferation of wild-type (WT), $\mathrm{KRAS}^{\mathrm{G} 12 \mathrm{D} /+}(\mathrm{K}), \mathrm{LKB1}^{-/-}(\mathrm{L})$ and $\mathrm{KRAS}^{\mathrm{G} 12 \mathrm{D} /+} ; \mathrm{LKB1}^{-/-}(\mathrm{KL})$ cells $(n=6)$. $\mathbf{k}-\mathbf{t}$, In vitro studies of K and KL cells. $\mathbf{k}$, Detection of GLUT1 (SLC2A1) by immunofluorescence (scale bar, $20 \mu \mathrm{m}$ ) or immunoblot. 2-(4-amidinophenyl)- $1 \mathrm{H}$-indole-6-carboxamidine (DAPI) was used to visualize nuclei. Actin was used as the loading control. For gel source images see Supplementary Data Fig. 1. 1, Steady-state ATP levels under nutrient-replete conditions measured by CellTiterGlo (Promega), normalized to cell number and expressed as relative to ATP levels in K cells $(n=4)$. $\mathbf{m}$, Intracellular levels of pyruvate, lactate, and TCA cycle metabolites as detected by GC-MS. Values are normalized to cell number. Data are expressed as relative to the levels in K cells ( $n=6$ biological replicates). $\mathbf{n}$, Three-day proliferation of cells in $25 \mathrm{mM}$ glucose or acutely switched to media with the indicated reduced glucose concentrations $(n=6)$. Data are expressed as relative to day 0 . $\mathbf{0}-\mathbf{q}$, Proliferation of cells treated with $5 \mathrm{mM}$ 2-deoxyglucose (2DG) (o), $5 \mathrm{mM}$ dichloroacetate (DCA) (p) or $20 \mu \mathrm{M}$ galloflavin (gallo) (q). Values are expressed as percentage of normal growth (2DG $n=6$, DCA $n=8$, gallo $n=6$ ). $\mathbf{r}-\mathbf{t}, \mathrm{WT}, \mathrm{K}, \mathrm{L}$ and $\mathrm{KL}$ cells were measured for glucose uptake using $2 \mathrm{NBDG}(\mathbf{r}, n=6)$, lactate release into the medium $(\mathbf{s}, n=4)$, and expression of glycolytic genes $(\mathbf{t}, n=6)$. Data are pooled from two $(\mathbf{j}, \mathbf{l}, \mathbf{n}-\mathbf{r})$ or three $(\mathbf{t})$ experiments or representative of two $(\mathbf{h}, \mathbf{i}, \mathbf{s})$ experiments. For all panels, error bars show s.d. unless otherwise stated; $* P<0.05$, $* * P<0.01$, $* * * P<0.001$. 
a

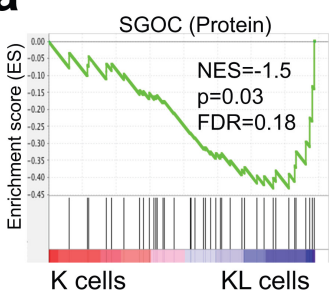

f

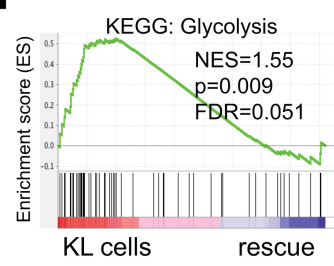

b

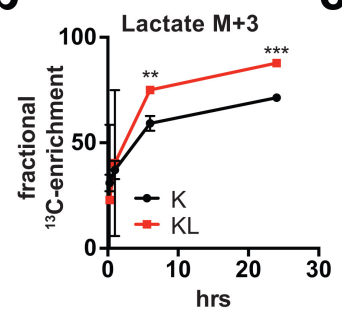

C

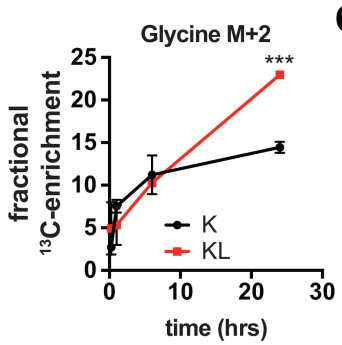

KEGG: Glycine/Serine/Threonine

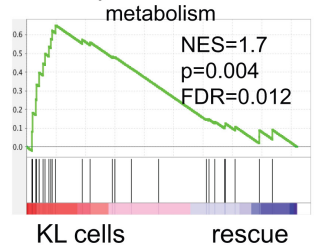

KEGG: One-carbon pool KEGG: Folate Biosynthesis

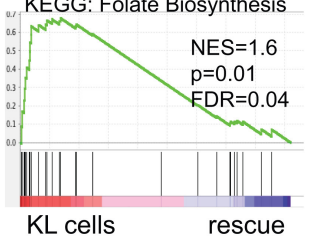

d

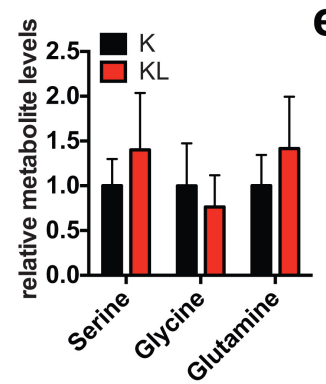

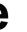

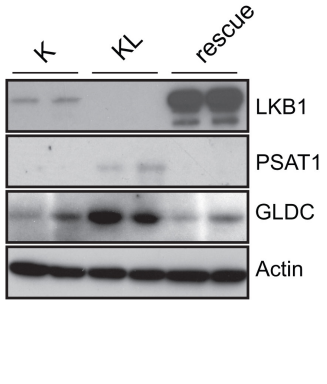

g

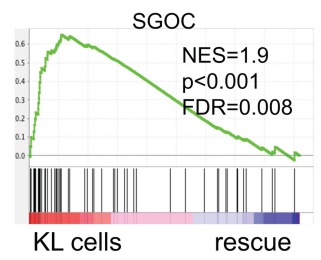

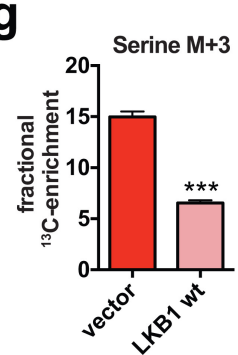

Extended Data Figure $2 \mid$ LKB1 loss induces the serine-glycine-onecarbon network. a, GSEA showing enrichment of proteins involved in the serine-glycine-one-carbon network ${ }^{18}$ in KL cells compared to K cells using global proteomics ( $n=4$ samples per group). $\mathbf{b}$, Plot of isotopomer abundance of $\mathrm{U}\left[{ }^{13} \mathrm{C}\right]$ glucose-derived intracellular $\mathrm{M}+3$ lactate over time ( $n=3$ independent biological replicates). c, Plot of isotopomer abundance of $\mathrm{U}\left[{ }^{13} \mathrm{C}\right]$ glucose-derived intracellular $\mathrm{M}+2$ glycine over time $(n=3$ independent biological replicates). d, Intracellular levels of serine, glycine and glutamine as detected by GC-MS. Values normalized to cell number. Data are expressed as relative to the levels in K cells ( $n=6$ biological replicates). e, PSAT1 and GLDC expression determined by immunoblot in $\mathrm{K}$ and $\mathrm{KL}$ cells and in KL cells transduced with LKB1 cDNA. Actin was used as loading control. For gel source images see Supplementary Data Fig. 1. f, GSEA of RNA-seq data showing suppression of genes involved in glycolysis, serine biosynthesis, folate cycle and the serine-glycine-onecarbon network upon re-expression of LKB1 cDNA in KL cells (rescue, $n=2$ samples) compared to parental KL cells ( $n=4$ samples). g, Plot of isotopomer abundance of $\mathrm{U}\left[{ }^{13} \mathrm{C}\right]$ glucose-derived intracellular $\mathrm{M}+3$ serine $6 \mathrm{~h}$ after addition of $\mathrm{U}\left[{ }^{13} \mathrm{C}\right]$ glucose $(n=3$ independent biological replicates). For all panels, error bars show s.d. unless otherwise stated; $* P<0.05, * * P<0.01, * * * P<0.001$. 
a

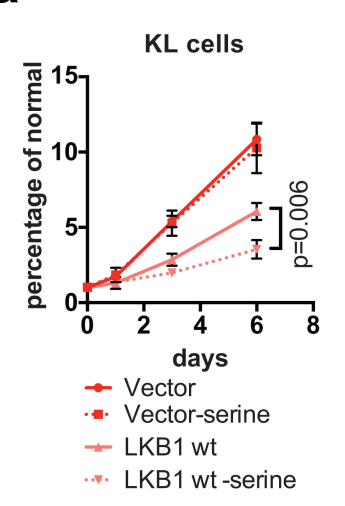

e

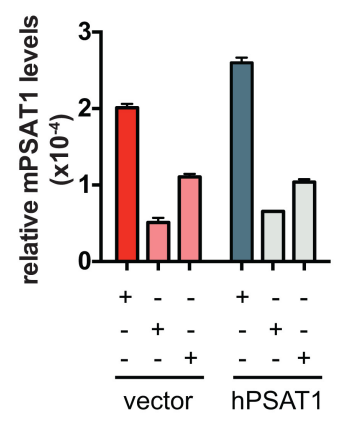

h

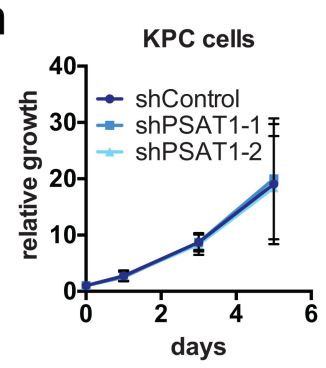

b
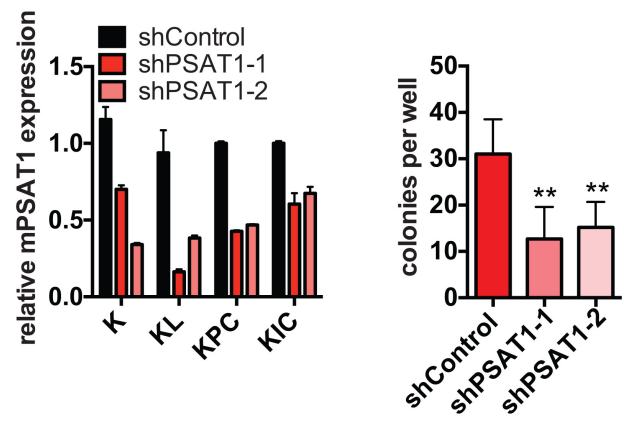

f
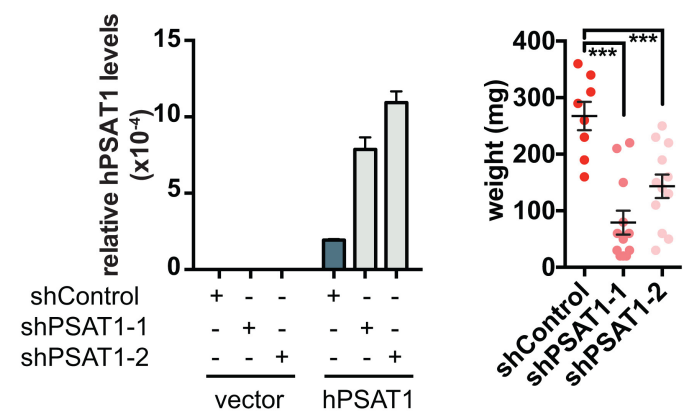

Growth in $0 \mathrm{mM}$ serine vs $0.4 \mathrm{mM}$ serine

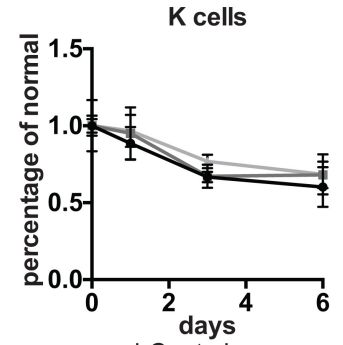

$\rightarrow$ shControl

- shPSAT1-1

- shPSAT1-2

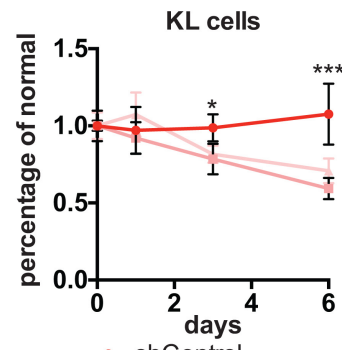

$\rightarrow$ shControl

- shPSAT1-1

- shPSAT1-2

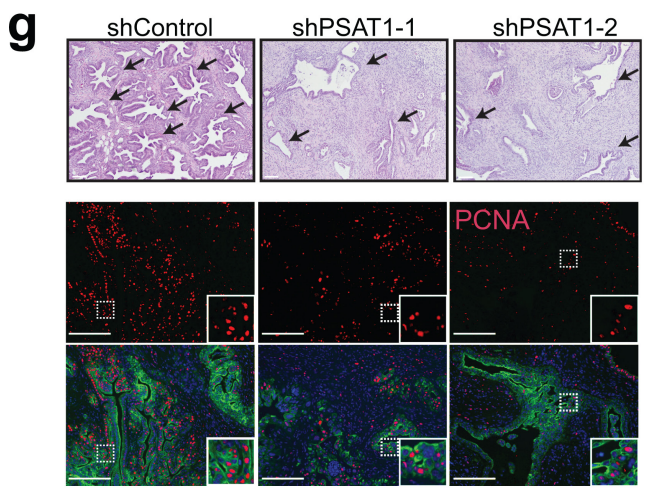

DAPI PCNA CK19

j
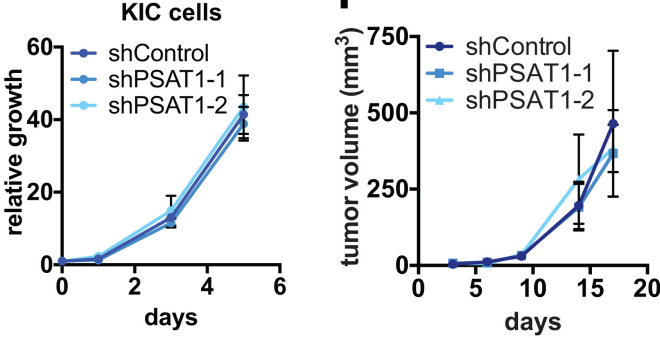

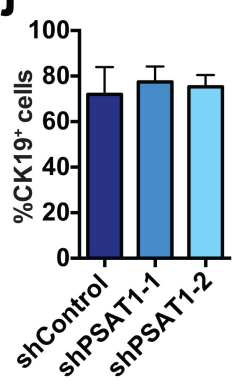

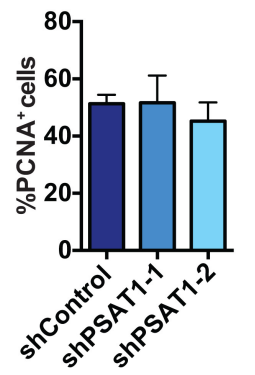

k
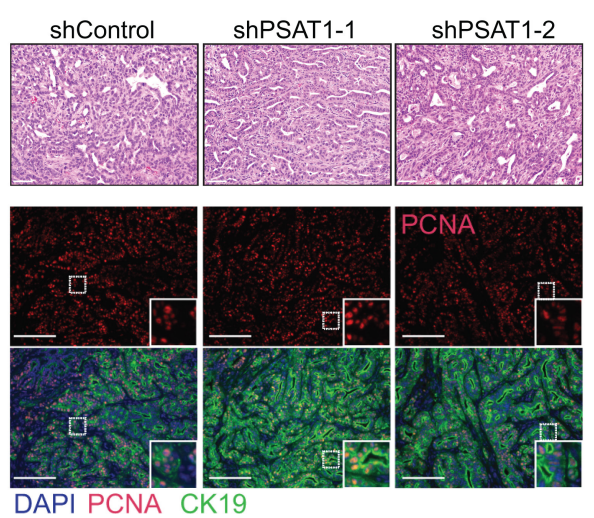

Extended Data Figure 3 | See next page for caption. 
Extended Data Figure 3 | The de novo serine biosynthesis pathway is required for KL but not KPC tumour growth. a, Proliferation of KL cells transduced with vector or LKB1 cDNA and cultured in the presence or absence of $0.4 \mathrm{mM}$ serine. Growth is expressed as relative to day 0 $(n=3)$. LKB1 re-expression slows growth and results in sensitivity to serine deprivation. $\mathbf{b}$, qRT-PCR showing effective knockdown of PSAT1 in K, KL, KPC and KIC cells transduced with shControl or two different shRNAs against PSAT1 $(n=2)$. Data are expressed as relative to shControl for each cell line. 18S rRNA was used for normalization. c, Number of colonies formed in soft agar by KL cells transduced with shControl or two different shRNAs against PSAT1 $(n=6)$. d, Proliferation of K or KL cells transduced with shControl or two independent shRNAs against PSAT1 in the absence of serine. Data are expressed as percentage of growth in the presence of $0.4 \mathrm{mM}$ serine $(n=6)$. e, qRT-PCR showing effective knockdown of endogenous mouse PSAT1 (mPSAT1, endogenous) and forced expression of human PSAT1 (hPSAT1, exogenous) in KL cells transduced with shControl, shPSAT1-1, shPSAT1-2, vector or hPSAT1 cDNA. Data are normalized to $18 \mathrm{~s}$ rRNA $(n=2)$. f, Weight at the time of harvesting of subcutaneous tumours from KL cells transduced with shControl $(n=8)$, shPSAT1-1 $(n=12)$, or shSPAT1-2 $(n=12)$. Error bars show s.e.m. g, Haematoxylin and eosin-stained sections and immunofluorescence analysis of representative tumours derived from subcutaneous injections of KL cells transduced with shControl,
shPSAT1-1, or shPSAT1-2. Note that PSAT1 knockdown tumours have a reduction in malignant glands (arrows) relative to the fibrotic stroma. Lower panels: anti-CK19 (green) was used to visualize the neoplastic epithelium and anti-PCNA (red) was used to mark proliferation. DAPI was used to stain nuclei (blue). h, Proliferation of KPC and KIC pancreatic cancer cells transduced with shControl or two independent shRNAs against PSAT1. Growth is expressed as relative to day 0 ( $n=6$ for KPC and $n=4$ for KIC). i-k, KPC cells were transduced with shControl $(n=6)$, shPSAT1-1 $(n=6)$ or shSPAT1-2 $(n=4)$ and injected into SCID mice. i, Volume (left) and weight (right) of subcutaneous tumours. Error bars show s.e.m. For source data on tumour volume, see Supplementary Data Table 4. $\mathbf{j}, \mathbf{k}$, Tumours in $\mathbf{i}$ were stained using anti-CK19 antibody (green) to visualize the neoplastic epithelium and anti-PCNA staining (red) to mark proliferating cells. DAPI was used to stain nuclei (blue). The proportion of stained CK $19^{+}$cells is quantified in $\mathbf{j}(n=4$, representative tumours) and $\mathrm{CK} 19^{+}$cells with nuclear PCNA staining are quantified in $\mathbf{j}$. There are no significant effects on any of these parameters. k, Haematoxylin and eosin-stained sections (top) and immunofluorescence analysis (bottom) of representative tumours. Scale bars, $100 \mu \mathrm{m}$. Insets show threefold magnification. Data pooled from two $(\mathbf{c}, \mathbf{d})$ or representative of two $(\mathbf{a}, \mathbf{b}, \mathbf{e})$ or three $(\mathbf{h})$ experiments. For all panels, error bars show s.d. unless otherwise stated; $* P<0.05$, $* * P<0.01, * * * P<0.001$ 


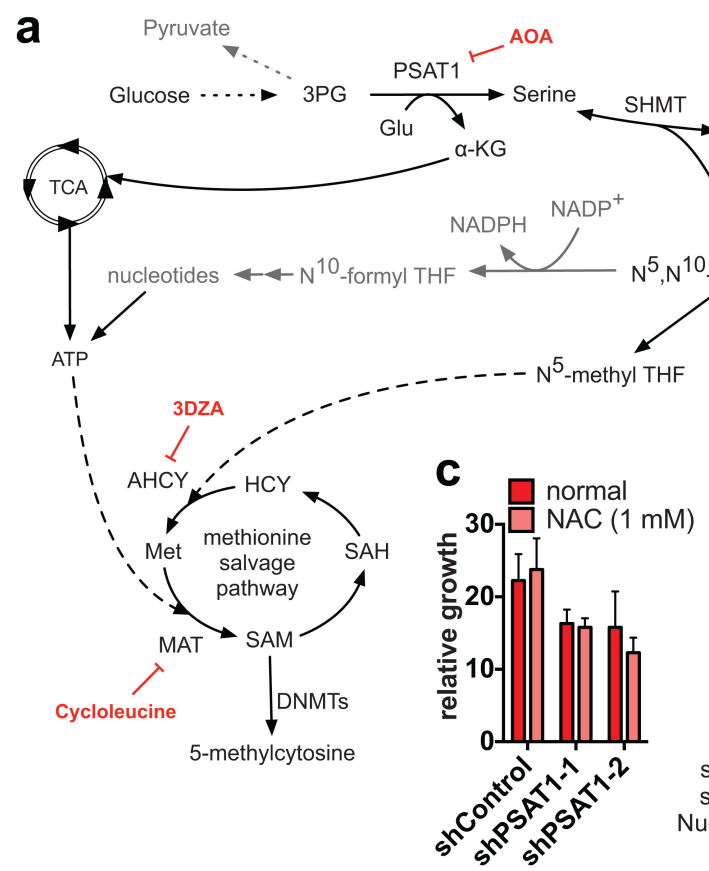

g

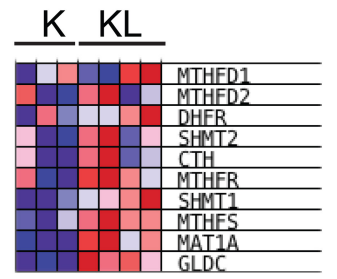

RNA

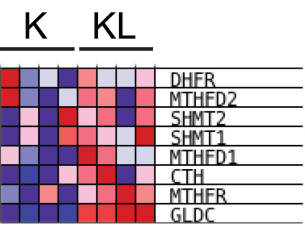

protein

Extended Data Figure 4 | Characterization of serine-glycine-onecarbon pathway in KL cells. a, Detailed graph of SGOC network. Enzymatic inhibitors used in this study are marked in red. b, ROS in K or KL cells transduced with shControl, shPSAT1-1 or shPSAT1-2 measured by DCFDA (left) and CellRox staining (right). Data are normalized to cell number $(n=4)$. c, Six-day proliferation assay of KL cells transduced with shControl, shPSAT1-1 or shPSAT1-2, showing lack of growth rescue by $\mathrm{N}$-acetylcysteine (NAC). Data are expressed as relative to day $0(n=6)$. d, Three-day growth assay of KL cells transduced with shControl, shPSAT1-1 or shPSAT1-2, showing the lack of rescue by excess nucleosides (adenosine, guanosine, thymidine, uridine, cytidine; $1 \mathrm{mM}$ each). Data are presented as percentage of the growth of shControl cells
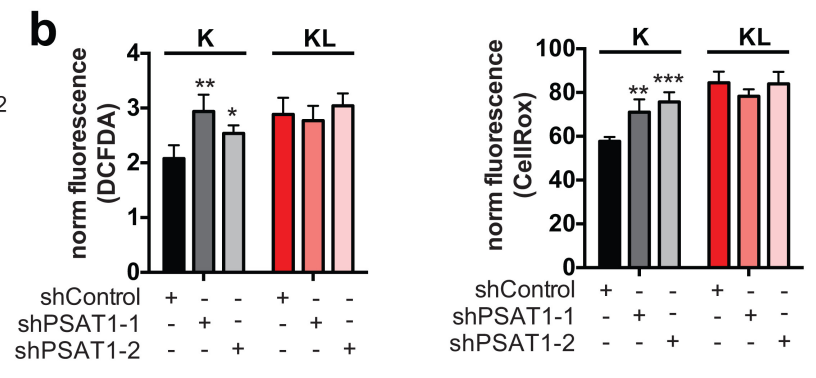

d
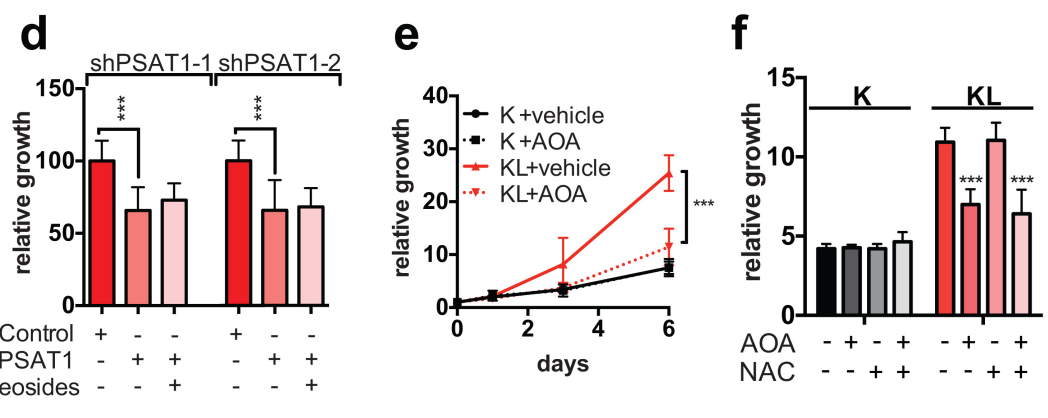

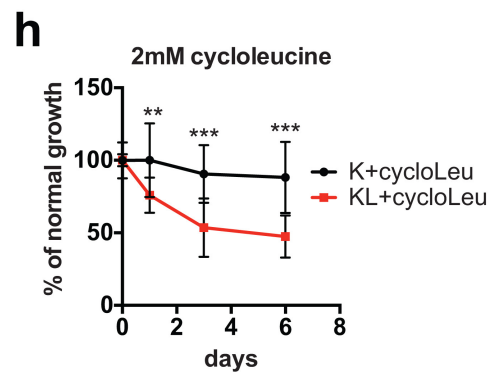

$(n=16)$. e, Proliferation of K or KL cells treated with aminooxyacetate (AOA). Data are expressed as relative to day $0(n=8)$. f, Five-day proliferation of K or KL cells treated with AOA and/or NAC. Data are expressed as relative to day $0(n=6)$. g, Data from RNA-seq (left) and quantitative proteomics (right) showing levels of genes involved in the production of SAM in K and KL. The data plotted are expressed as mean-centred values. h, Proliferation of K or KL cells treated with $2 \mathrm{mM}$ cycloleucine. Data are expressed as percentage of the growth of vehicle treated cells $(n=12)$. Data are pooled from two $(\mathbf{b}-\mathbf{f}, \mathbf{h})$ experiments. For all panels, error bars show s.d. unless otherwise stated; $* P<0.05$, $* * P<0.01, * * * P<0.001$. 
a

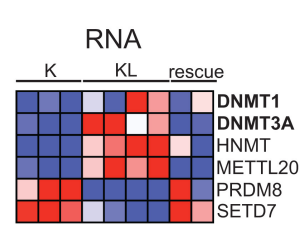

f

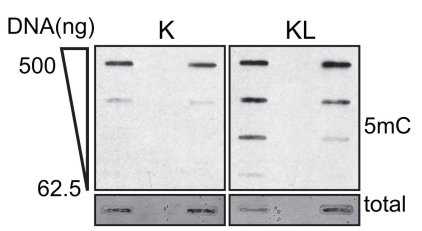

i

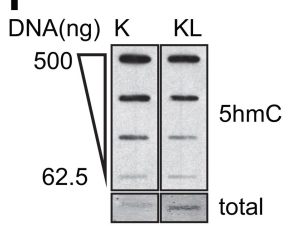

b

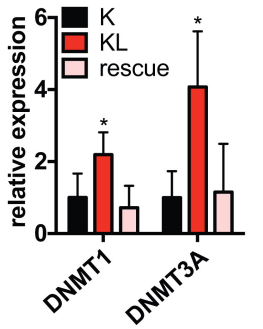

C
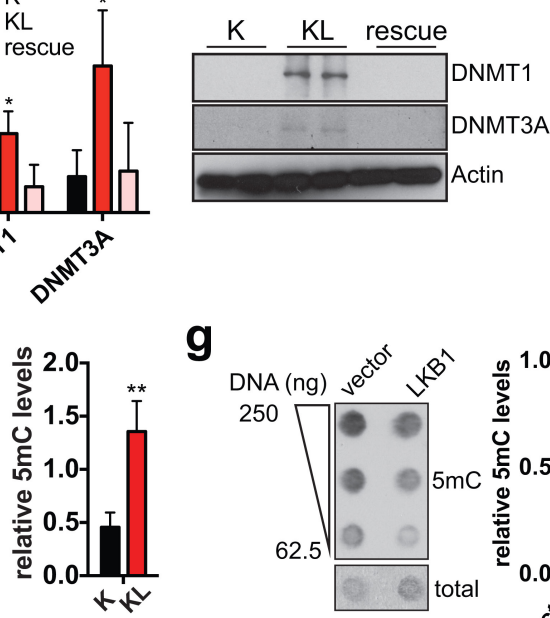

g

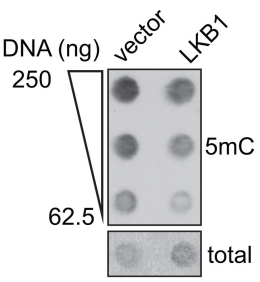

d

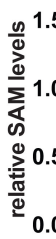

e

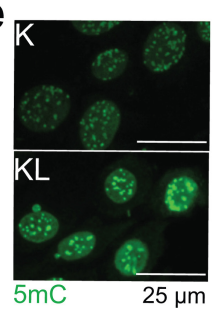

K vs KL

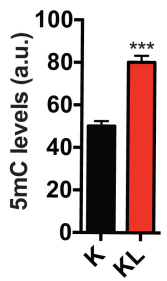

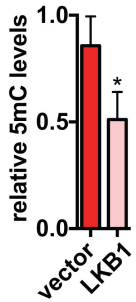

h

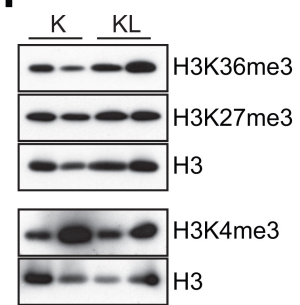

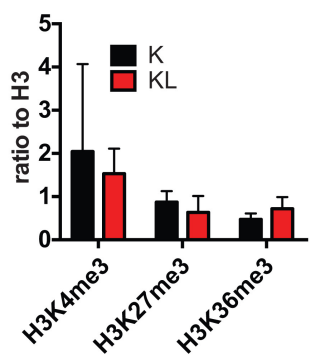

Extended Data Figure 5 | Deletion of LKB1 induces DNMT1 and DNMT3A expression and increases global DNA methylation. a, Heat map of RNA-seq data showing levels of the differentially regulated SAMusing enzymes. Plotted data are expressed as mean centred values. b, Expression of DNMT1 and DNMT3A in K, KL, and KL cells transduced with LKB1 cDNA (rescue) was measured by qRT-PCR. Levels were normalized to $18 \mathrm{~S}$ rRNA. Data are expressed as relative to K cells $(n=4$, representative of two experiments). c, Immunoblots of lysates from $\mathrm{K}$, KL or rescue cells were probed for DNMT1 or DNMT3A. Actin was used as loading control. For gel source images see Supplementary Data Fig. 1. d, Measurement of SAM in K and KL cells treated with 5-aza-2deoxycytidine (decitabine) or RG108 for 3 days. In each case, data are expressed as relative to the amount of SAM in vehicle-treated $\mathrm{K}$ cells, which was arbitrarily set to 1 ( $n=6$ independent replicates).

e, Immunofluorescence staining and quantitation of $5 \mathrm{mC}$ in $\mathrm{K}$ or $\mathrm{KL}$ cells
(77-130 cells). Scale bar, $25 \mu \mathrm{m}$. f, Dot blot of DNA isolated from K or KL cells probed with anti-5mC antibody. Quantified signal was normalized to total DNA as measured by methylene blue staining $(n=4$, independent replicates). $\mathbf{g}$, Dot blot of DNA isolated from KL cells transduced with empty vector or LKB1 cDNA probed with anti-5mC antibody. Quantified signal was normalized to total DNA as measured by methylene blue staining ( $n=3$, independent replicates). $\mathbf{h}$. Immunoblot analysis of histone 3 (H3) methyl marks from K or KL cells. Data are normalized to total H3 (K4me3, $n=2$; K27me3, $n=5$; K36me3, $n=5$, independent replicates). For gel source images see Supplementary Data Fig. 1. i, Dot blot of DNA isolated from $\mathrm{K}$ or KL cells probed with anti-5hmC antibody. Quantified signal was normalized to total DNA as measured by methylene blue staining ( $n=4$, independent replicates). For all panels, error bars show s.d. unless otherwise stated; $* P<0.05$, $* * P<0.01, * * * P<0.001$. 

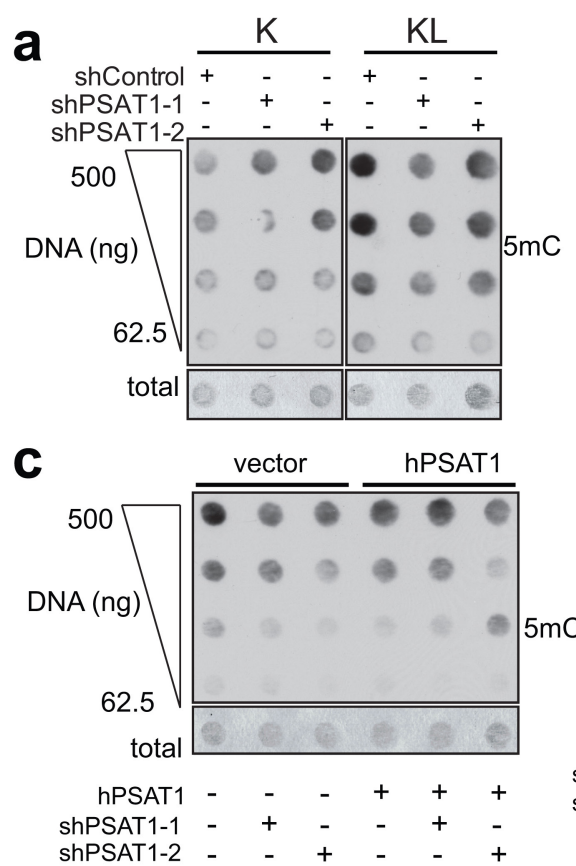

e
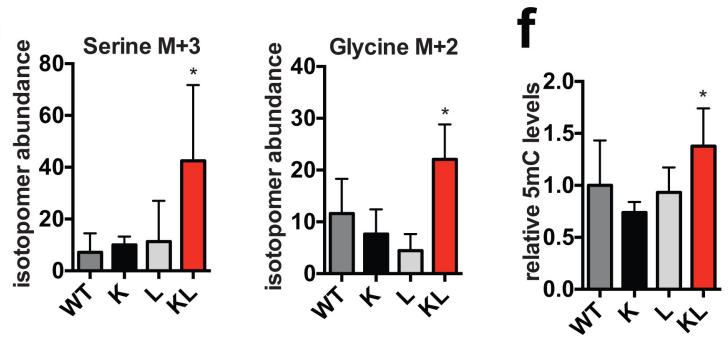

Extended Data Figure 6 | Serine pathway activity sustains DNA methylation in KL cells. a, Dot blot of DNA isolated from K or KL cells transduced with shControl, shPSAT1-1 or shPSAT1-2 probed with anti$5 \mathrm{mC}$ antibody. Total DNA was visualized by methylene blue staining. Graph shows quantified signal normalized to total DNA as measured by methylene blue staining (K cells, $n=4$; KL cells, $n=8$, independent replicates). b, Dot blot of DNA isolated from KPC cells transduced with shControl, shPSAT1-1 or shPSAT1-2 probed with anti-5mC antibody. The graph shows quantified signal normalized to total DNA $(n=4$, independent replicates). c, Dot blot of DNA probed with anti-5mC antibody. DNA was isolated from KL cells first transduced with vector or human PSAT1 then transduced with shControl, shPSAT1-1 or shPSAT-2. Graph shows quantified signal normalized to total DNA as measured by b

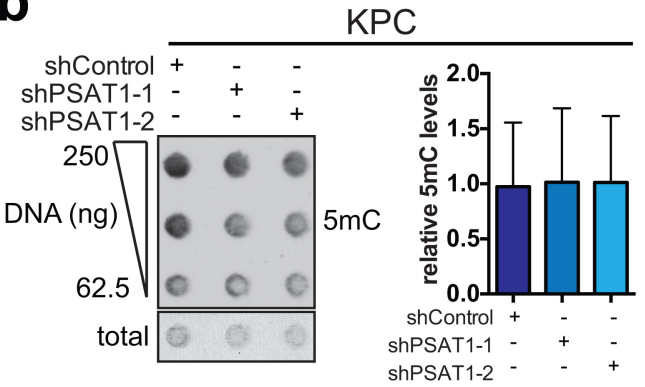

d

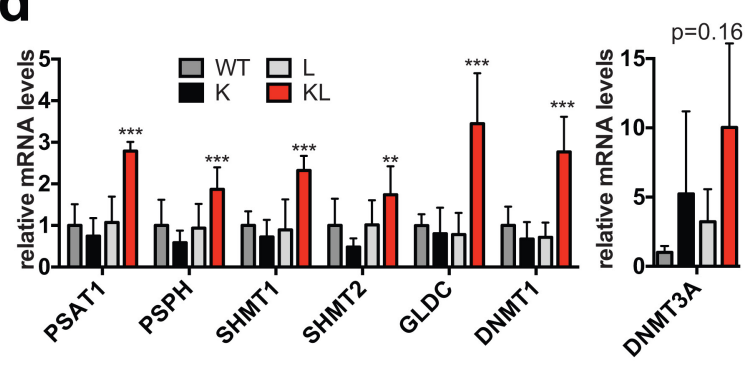

g

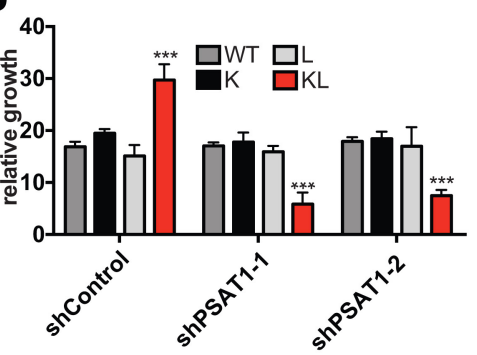

methylene blue staining ( $n=3$, independent replicates). d, Expression of serine pathway genes and DNMT genes in WT, K, L or KL cells by qRT-PCR. Data are normalized to $18 \mathrm{~S}$ and expressed as relative to $\mathrm{K}$ cells ( $n=6$, pooled data from two experiments). e, Plots of isotopomer abundance of $\mathrm{U}\left[{ }^{13} \mathrm{C}\right]$ glucose-derived $\mathrm{M}+3$ serine and $\mathrm{M}+2$ glycine, $6 \mathrm{~h}$ after addition of $\mathrm{U}\left[{ }^{13} \mathrm{C}\right]$ glucose ( $n=6$ independent biological replicates). f, Quantified DNA dot blot signal of DNA isolated from WT, K, L or $\mathrm{KL}$ cells probed with anti-5mC antibody normalized to total DNA as measured by methylene blue staining ( $n=4$, independent replicates). g, Five-day growth of WT, K, L or KL cells transduced with shControl or two shRNAs against PSAT1. Data are expressed as relative to day $0(n=4$, pooled from two experiments). For all panels, error bars show s.d. unless otherwise stated; $* P<0.05, * * P<0.01$, *** $P<0.001$. 

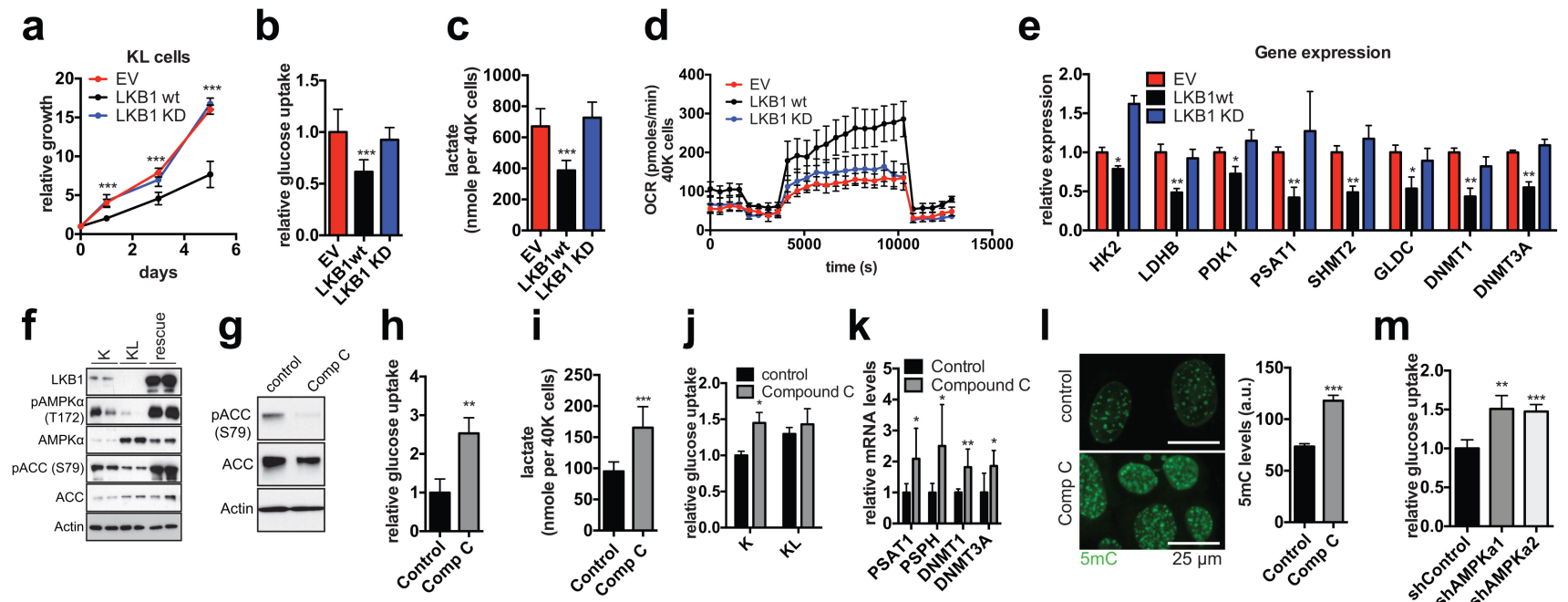

m
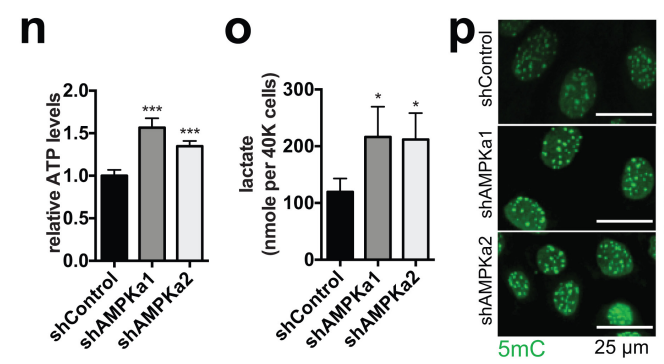

q
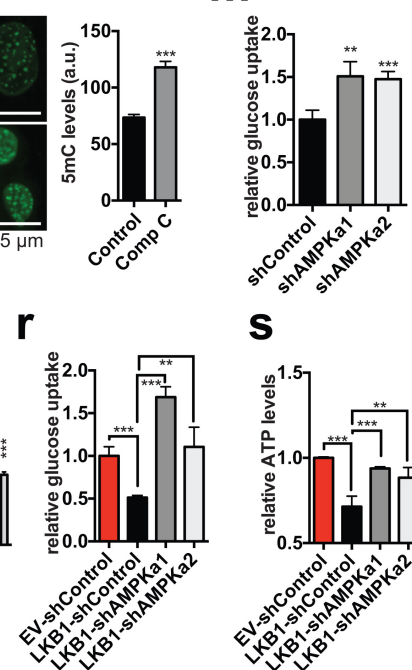

5
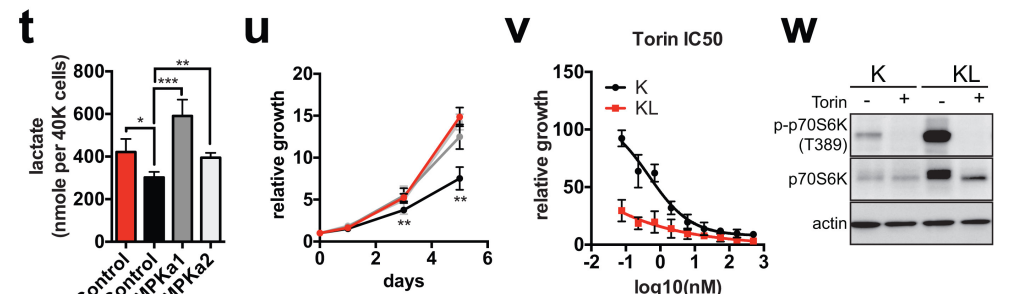

$$
\begin{aligned}
& \rightarrow \text { EV-shControl } \\
& \rightarrow \text { LKB1-shControl } \\
& \rightarrow \text { LKB1-shAMPKa1 } \\
& \rightarrow \text { LKB1-shAMPKa2 }
\end{aligned}
$$
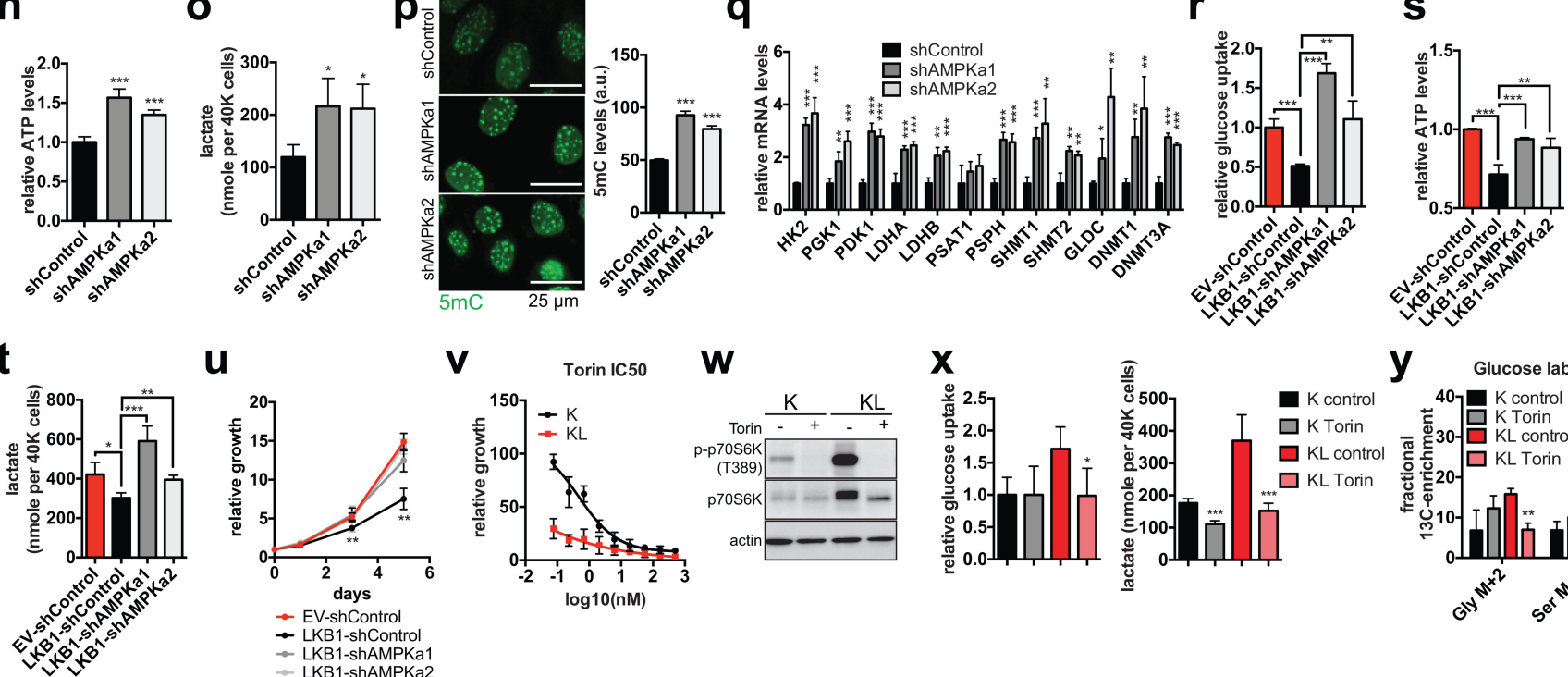

rate

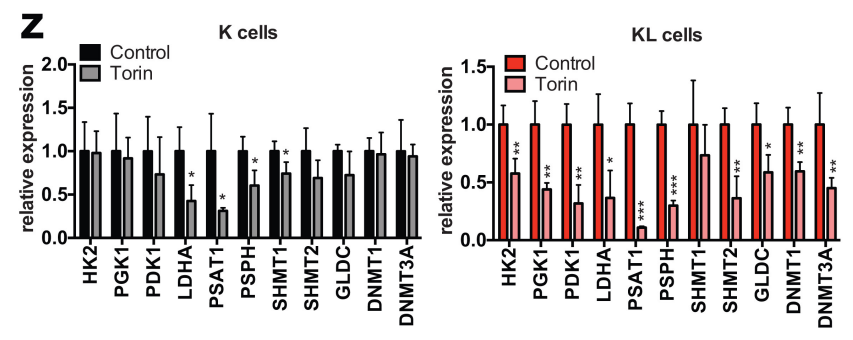

Extended Data Figure 7 | See next page for cation. 
Extended Data Figure 7 | LKB1-mediated regulation of glycolysisSGOC-DNMT pathway involves the AMKP-mTOR axis. a-e, KL cells were transduced with vector, wild-type LKB1 or kinase-dead LKB1. a, Proliferation, expressed as relative to day $0(n=4)$. b. Glucose uptake measured using 2NBDG followed by fluorimetry (data normalized to cell number and expressed as relative to KL-vector cells $(n=8)$ ). c, Lactate levels measured by fluorimetry $3 \mathrm{~h}$ after medium change, normalized to cell number $(n=8)$. $\mathbf{d}$, Oxygen consumption rates measured in normal duct medium, followed by injections with $4 \mu \mathrm{M}$ oligomycin $(\mathrm{O}), 4 \mu \mathrm{M}$ FCCP (F), or $4 \mu \mathrm{M}$ antimycin A (A) $(n=3)$. e, Expression of the indicated genes measured by qRT-PCR $(n=4)$, with levels normalized to $18 \mathrm{~S}$ rRNA. Data are expressed as relative to KL-empty vector (EV) cells. f, Immunoblot of K, KL or KL cells transduced with LKB1 cDNA (rescue). For gel source images see Supplementary Data Fig. 1. g, Immunoblot of K cells treated overnight with $10 \mu \mathrm{M}$ Compound C. For gel source images see Supplementary Data Fig. 1. h, i, Glucose uptake (h) and $3 \mathrm{~h}$ lactate production (i) in the same cells as in $\mathbf{h}$. Data are normalized to cell number and expressed as relative to control $\mathrm{K}$ cells (glucose uptake, $n=3$; lactate, $n=4)$ ). $\mathbf{j}$, Glucose uptake in K or KL cells treated with vehicle or $10 \mu \mathrm{M}$ Compound C. Data are normalized to cell number and expressed as relative to control K cells $(n=4)$. Note the blunted response of KL cells. $\mathbf{k}$, qRT-PCR analysis of the indicated genes in the same cells as in $\mathbf{h}$. Levels are normalized to $18 \mathrm{~S}$ rRNA. Data are expressed as relative to control $\mathrm{K}$ cells $(n=6)$. 1, Immunostaining for 5 - $\mathrm{mC}$ (left) and quantification of staining (right) in $\mathrm{K}$ cells treated with vehicle or Compound $\mathrm{C}$ for 4 days (158-163 cells). $\mathbf{m}-\mathbf{q}$, K cells were transduced with shControl or shRNAs against AMPKa1 or AMPKa2. m, Glucose uptake. Data are normalized to cell number and expressed as relative to shControl-treated cells $(n=4)$. n, Steady-state ATP levels measured with CellTiterGlo (Promega), normalized to cell number and expressed as relative to ATP levels in shControl-treated cells $(n=4)$. o, Lactate levels measured by fluorimetry $3 \mathrm{~h}$ after medium change. Data are normalized to cell number $(n=3)$. p, Immunostaining for 5-mC (left) and quantification of staining (right) (159-296 cells). q, Expression of the indicated genes determined by qRT-PCR. Levels are normalized to $18 \mathrm{~S}$ rRNA. Data are expressed as relative to shControl cells $(n=4)$. $\mathbf{r}-\mathbf{u}$, KL cells were transduced with vector or an LKB1 cDNA and shControl or shRNAs against AMPKa1 or AMPKa2. r, Glucose uptake. Data are normalized to cell number and expressed as relative to vector-shControl cells $(n=4)$. $\mathbf{s}$, Steady-state ATP levels normalized to cell number and expressed as relative to ATP levels in EV-shControl cells $(n=4)$. t, Lactate levels $3 \mathrm{~h}$ after medium change. Data are normalized to cell number $(n=4)$. $\mathbf{u}$, Proliferation expressed as relative to day $0(n=3)$. v, Impact of Torin 1 treatment on growth of K and KL cells $(n=4)$. w, Immunoblot of K and KL cells treated with vehicle or $25 \mathrm{nM}$ Torin 1. For gel source images see Supplementary Data Fig. 1. x, Glucose uptake and $3 \mathrm{~h}$ lactate production in K or KL cells treated overnight with $25 \mathrm{nM}$ Torin 1 . Data are normalized to cell number $(n=4)$. y, Isotopomer abundance of $\mathrm{U}\left[{ }^{13} \mathrm{C}\right]$ glucose-derived serine and glycine in the same cells as in $\mathbf{r}$ ( $n=3$ independent replicates). Cells were labelled with $\mathrm{U}\left[{ }^{13} \mathrm{C}\right]$ glucose for $6 \mathrm{~h}$. $\mathbf{z}$, Expression of the indicated genes in Torin-treated $\mathrm{K}$ (left) or KL cells (right) determined by qRT-PCR. Levels are normalized to $18 \mathrm{~S}$ rRNA. Data are expressed as relative to control-treated cells $(n=4)$. Data are pooled from two $(\mathbf{b}, \mathbf{c}, \mathbf{e}, \mathbf{i}-\mathbf{n}, \mathbf{p}-\mathbf{t}, \mathbf{v}, \mathbf{x}, \mathbf{z})$ or representative of two $(\mathbf{a}, \mathbf{d}, \mathbf{h}, \mathbf{o})$ or three $(\mathbf{u})$ experiments. Error bars show s.e.m. in d, s.d. in all other panels. $* P<0.05, * * P<0.01, * * * P<0.001$. 


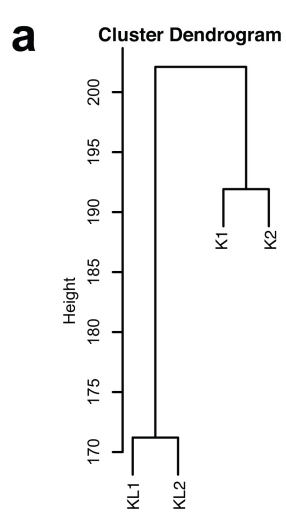

b

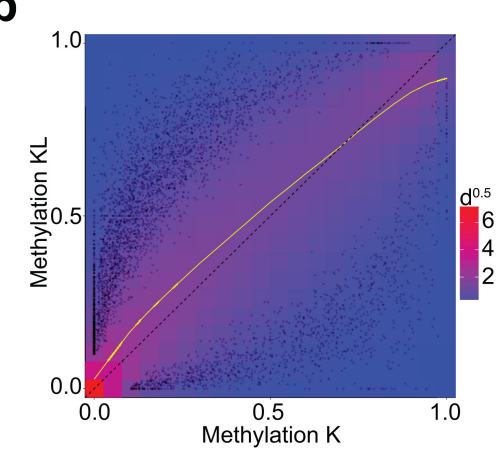

c

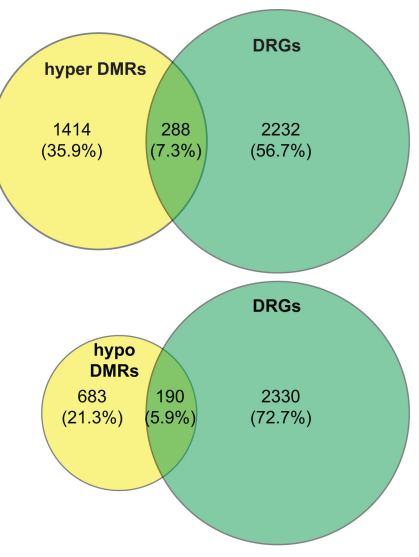

d

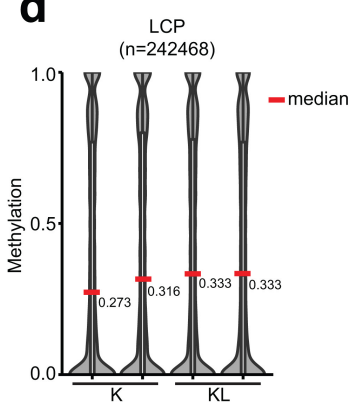

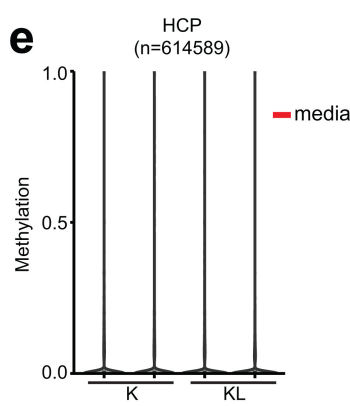
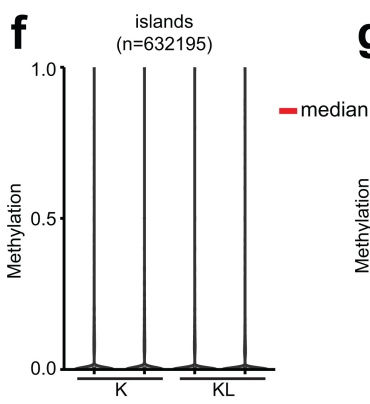

g $\underset{\substack{\text { shores } \\(n=242468)}}{2}$
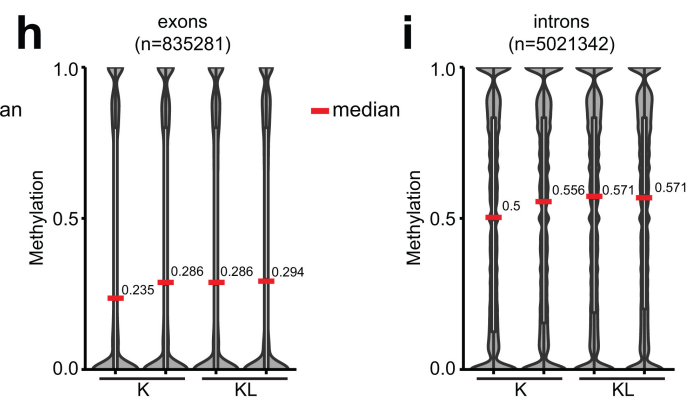

j

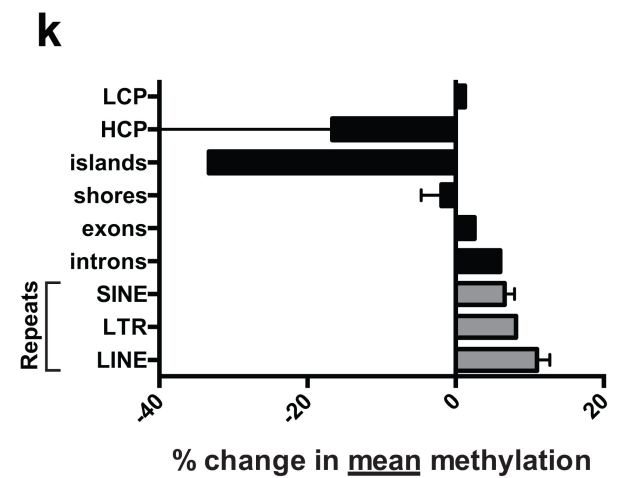

m

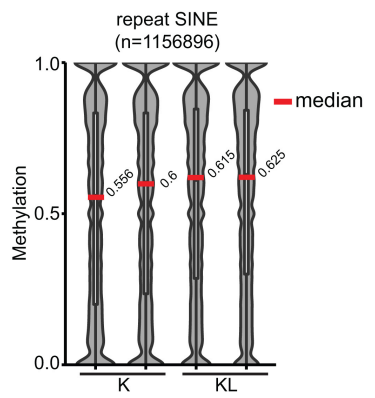

\section{I} Enrichment of retrotransposons in

\begin{tabular}{|c|c|c|c|c|}
\multicolumn{5}{c|}{ association with DRGs } \\
$\begin{array}{c}\text { gene } \\
\text { bodies }\end{array}$ & $\begin{array}{c}\text { with } \\
\text { repeats }\end{array}$ & total & $\%$ & $\begin{array}{c}\text { chi } \text { yates } \\
\text { correction }\end{array}$ \\
\hline DRGs & 2262 & 2443 & $\mathbf{9 2 . 6 \%}$ & \multirow{2}{*}{$<0.0001$} \\
\hline genome & 18865 & 23355 & $\mathbf{8 0 . 7 \%}$ & \\
\hline
\end{tabular}

\begin{tabular}{|c|c|c|c|c|}
\hline $\begin{array}{c}5 k \boldsymbol{k} b \\
\text { promoters }\end{array}$ & $\begin{array}{c}\text { with } \\
\text { repeats }\end{array}$ & total & $\%$ & $\begin{array}{c}\text { chi }^{2} \text { yates } \\
\text { correction }\end{array}$ \\
\cline { 1 - 4 } DRGs & 2392 & 2443 & $\mathbf{9 7 . 9 \%}$ & \multirow{2}{*}{0.08} \\
\hline genome & 22722 & 23355 & $\mathbf{9 7 . 3 \%}$ & \\
\hline
\end{tabular}

\begin{tabular}{|c|c|c|c|c|}
\hline 3'UTR & $\begin{array}{c}\text { with } \\
\text { repeats }\end{array}$ & total & $\%$ & $\begin{array}{c}\text { chi }^{2} \text { yates } \\
\text { correction }\end{array}$ \\
\hline DRGs & 1270 & 4706 & $\mathbf{2 7 \%}$ & \multirow{2}{*}{0.08} \\
\hline genome & 10693 & 40213 & $\mathbf{2 6 . 6} \%$ & \\
\hline
\end{tabular}
retrotransposons in association with DRGs

\begin{tabular}{|c|c|c|c|c|}
\hline ㄴINE & $\begin{array}{l}\text { with } \\
\text { repeats }\end{array}$ & total & $\%$ & $\begin{array}{l}\text { chi }^{2} \text { yates } \\
\text { correction }\end{array}$ \\
\hline DRGs & 1819 & 2443 & $74.4 \%$ & \multirow{2}{*}{$<0.0001$} \\
\hline genome & 14448 & 23355 & $61.9 \%$ & \\
\hline SINE & $\begin{array}{l}\text { with } \\
\text { repeats }\end{array}$ & total & $\%$ & $\begin{array}{l}\text { chi }^{2} \text { yates } \\
\text { correction }\end{array}$ \\
\hline DRGs & 2231 & 2443 & $91.3 \%$ & \multirow{2}{*}{$<0.0001$} \\
\hline genome & 18445 & 23355 & $79 \%$ & \\
\hline LTR & $\begin{array}{l}\text { with } \\
\text { repeats }\end{array}$ & total & $\%$ & $\begin{array}{l}\text { chi }{ }^{2} \text { yates } \\
\text { correction }\end{array}$ \\
\hline DRGs & 1858 & 2443 & $76 \%$ & \multirow{2}{*}{$<0.0001$} \\
\hline genome & 15237 & 23355 & $65.2 \%$ & \\
\hline
\end{tabular}

Extended Data Figure 8 | See next page for caption. 


\section{RESEARCH ARTICLE}

Extended Data Figure 8 Loss of LKB1 increases DNA methylation in retrotransposon elements. a, Hierarchical clustering of $\mathrm{K}$ and $\mathrm{KL}$ samples based on methylation levels at 100-bp autosomal genomic tiles as measured by whole-genome bisulfite sequencing. $\mathbf{b}$, Two-dimensional density plot for methylation at non-repetitive 100-bp autosomal genomic tiles in KL versus K cells. The yellow line shows the mean methylation of tiles. The dots represent differentially methylated tiles (FDR $q$-value $<0.05$, methylation change $>0.1$ ). There are 3,395 hypermethylated tiles and 1,270 hypomethylated tiles in KL cells versus K cells. c, Overlap of genes associated with differentially methylated regions and differentially regulated genes (hyper DMRs, hypermethylated regions; hypo DMRs, hypomethylated regions; DRGs, differentially regulated genes). $\mathbf{d}-\mathbf{j}$, Distribution of methylation density within the low-CpG-promoters (LCPs), high-CpG-promoters (HCPs), islands, shores, exons, introns and LTRs. Numbers reflect median values. k, Average percentile change in methylation in the same elements as in $\mathbf{d}-\mathbf{j}$ as well as LINEs and LTRs. 1, Number of genes containing retrotransposon repeat elements (sum of LINEs, SINEs, LTRs) among the set of differentially regulated genes (DRGs) in K versus KL cells and among all genes. Note that the DRGs are enriched for the presence of retrotransposons in their gene bodies (top), but not in their promoters (middle) or $3^{\prime}$ UTRs (bottom). $\mathbf{m}$, Specific enrichment of LINE, SINE and LTR elements in the gene bodies of DRGs when compared to all genes in the genome. 

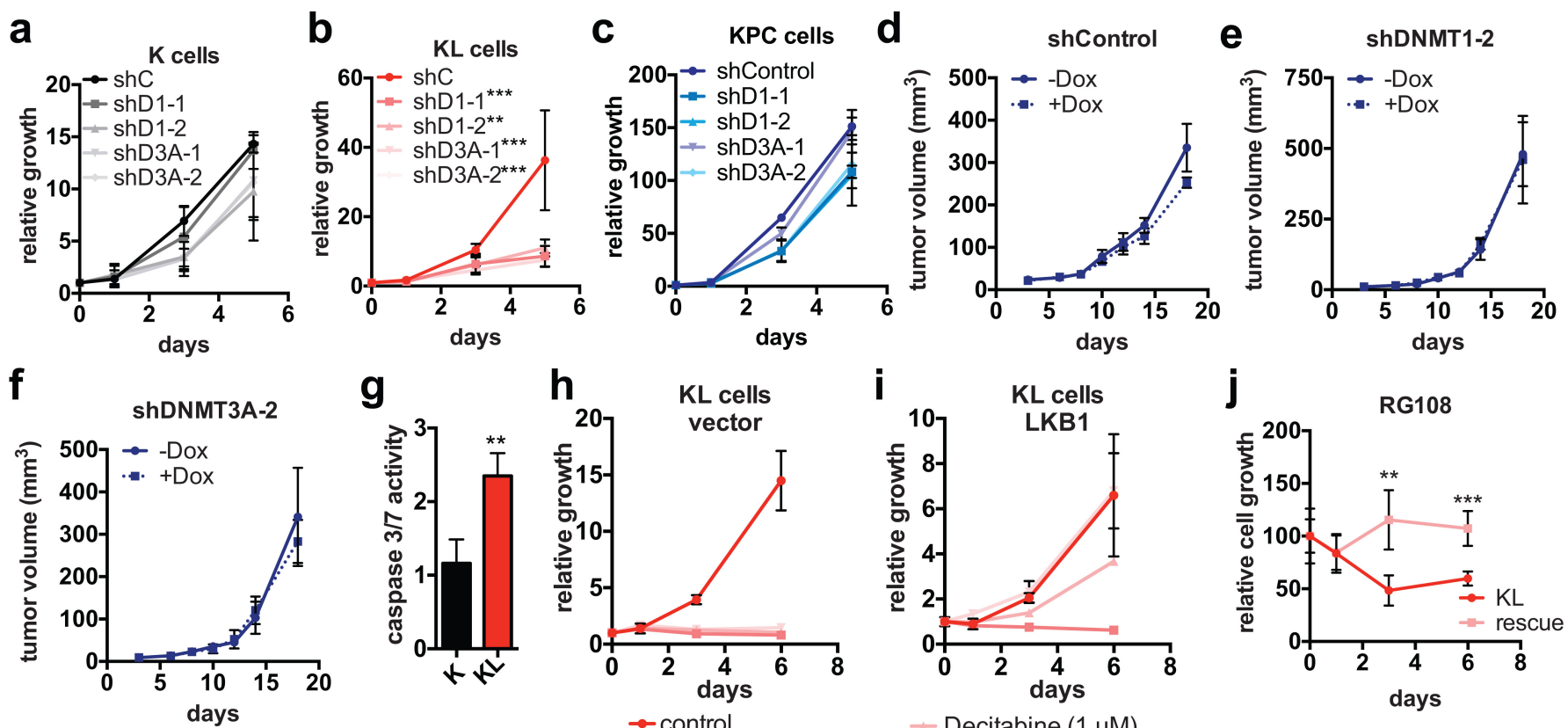

$$
\begin{aligned}
& \text { - control } \\
& \text { - Decitabine (10 uM) }
\end{aligned}
$$

- Decitabine (1 uM)
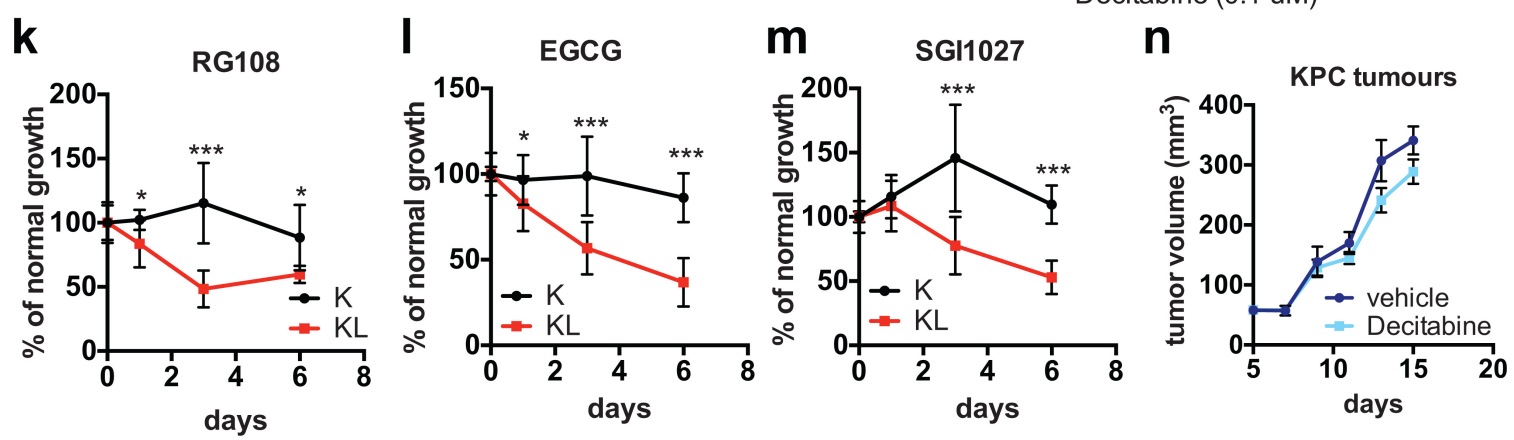

(
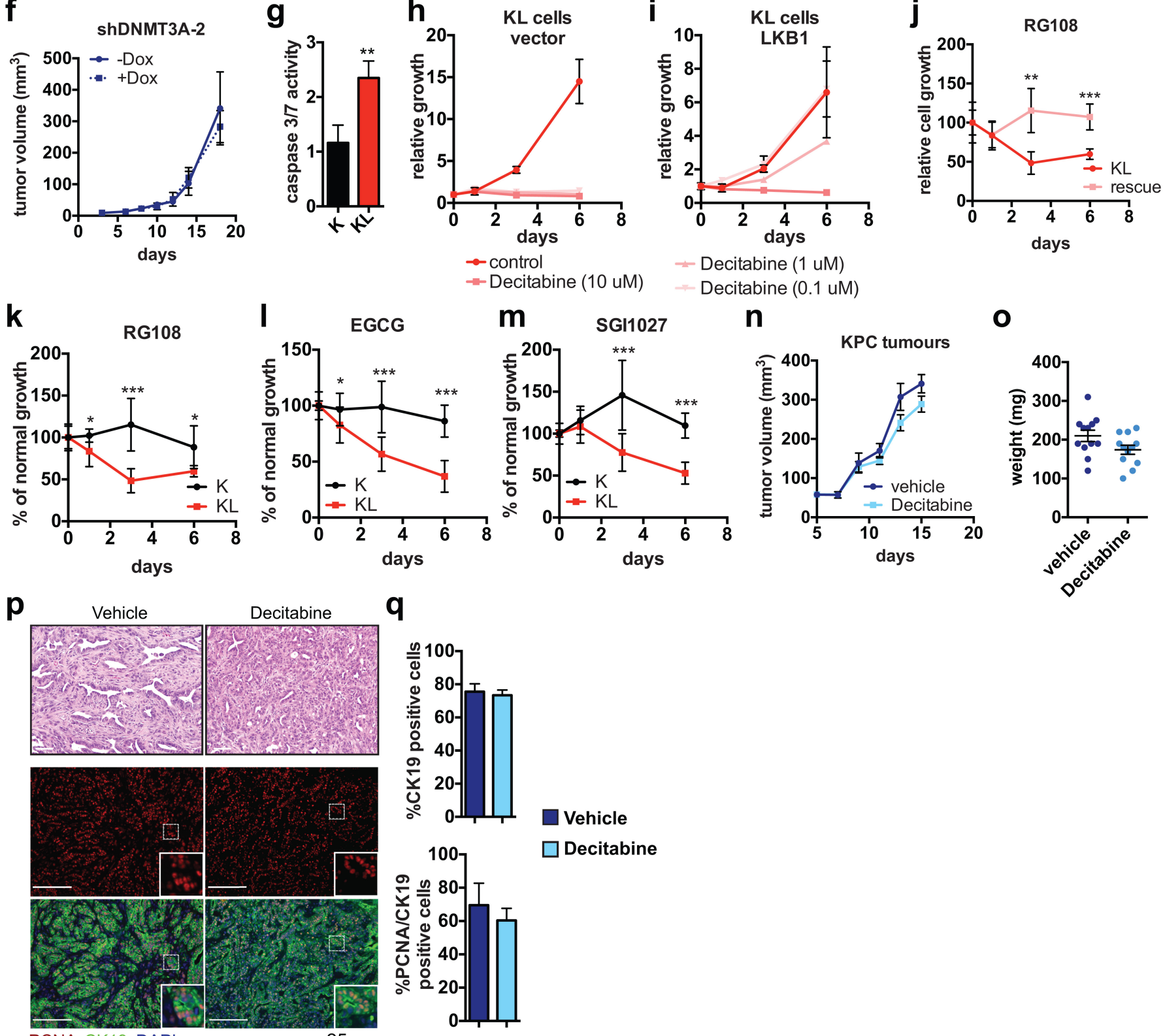

PCNA CK19 DAPI

$25 \mu \mathrm{m}$

Vehicle

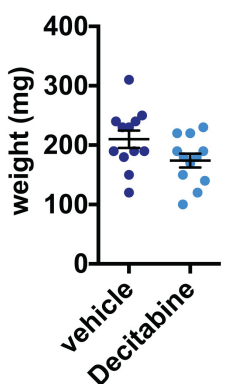

Extended Data Figure 9 | See next page for caption. 


\section{RESEARCH ARTICLE}

Extended Data Figure 9 | LKB1 deficiency confers hypersensitivity to inhibitors of DNA methylation. a-c, Proliferation of K (a), KL (b) and KPC (c) cells transduced with shControl or two shRNAs against each of DNMT1 (D1) or DNMT3A (D3A). Data are expressed as relative to day 0 (K and KL $n=6$, KPC $n=4)$. d-f, Volume of subcutaneous tumours derived from KPC cells transduced with doxcycline (Dox)-inducible shRNAs against DNMT1 or DNMT3A $(n=4)$. Doxcycline was introduced to the drinking water when tumours reached $125 \mathrm{~mm}^{3}$. Error bars show s.e.m. For source data on tumour volume, see Supplementary Data Table 4. g, Apoptosis measured by caspase 3/7 activity in K or KL cells treated with decitabine for $48 \mathrm{~h}$. Values are normalized to cell number $(n=3)$. $\mathbf{h}, \mathbf{i}$, Proliferation of KL cells transduced with empty vector (h) or LKB1 cDNA (i) treated with decitabine. Data are expressed as relative to day 0 $(n=3)$. j, Proliferation of KL cells transduced with empty vector or LKB1 cDNA treated with RG108. Data are expressed as percentage of growth of untreated cells $(n=6) . \mathbf{k}-\mathbf{m}$, Proliferation of K or KL cells treated with
RG108 $(n=6)(\mathbf{k})$, EGCG $(n=12)(\mathbf{l})$ or SGI1027 $(n=12)(\mathbf{m})$. Data are expressed as percentage of growth of untreated cells. $\mathbf{n}-\mathbf{q}$, Mice bearing subcutaneous KPC tumours were treated with decitabine $(n=12)$ or vehicle $(n=12)$ when tumours reached $125 \mathrm{~mm}^{3}$. n, o, Tumour volume (n) and final tumour weight (o). Error bars show s.e.m. For source data on tumour volume, see Supplementary Data Table 4. p, Haematoxylin and eosin-stained slides from representative tumours (top). Bottom, anti-CK19 (green) was used to visualize the neoplastic epithelium and anti-PCNA (red) was used to mark proliferating cells. DAPI was used to stain nuclei (blue). q, Quantification of the CK19 ${ }^{+}$neoplastic epithelial compartment (\%CK19 $9^{+}$cells/total cells; top). Quantification of CK19 $9^{+}$cells with nuclear PCNA staining (bottom; $n=6$ ). Scale bars, $100 \mu \mathrm{m}$. Insets are threefold magnification. Data pooled from two $(\mathbf{a}-\mathbf{c}, \mathbf{j})$ or four $(\mathbf{k}-\mathbf{m})$ experiments or representative of two (h) or three (g) experiments. Error bars show s.d. unless otherwise stated; $* P<0.05, * * P<0.01, * * * P<0.001$. 
a

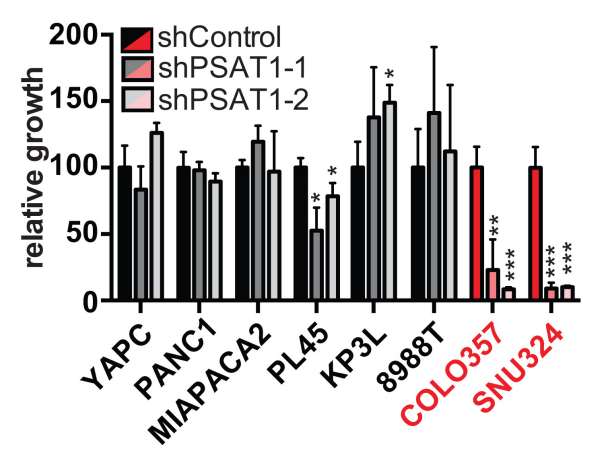

b

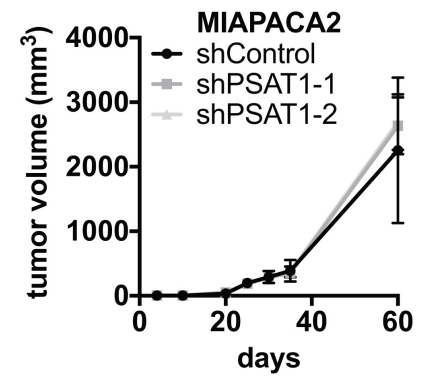

C
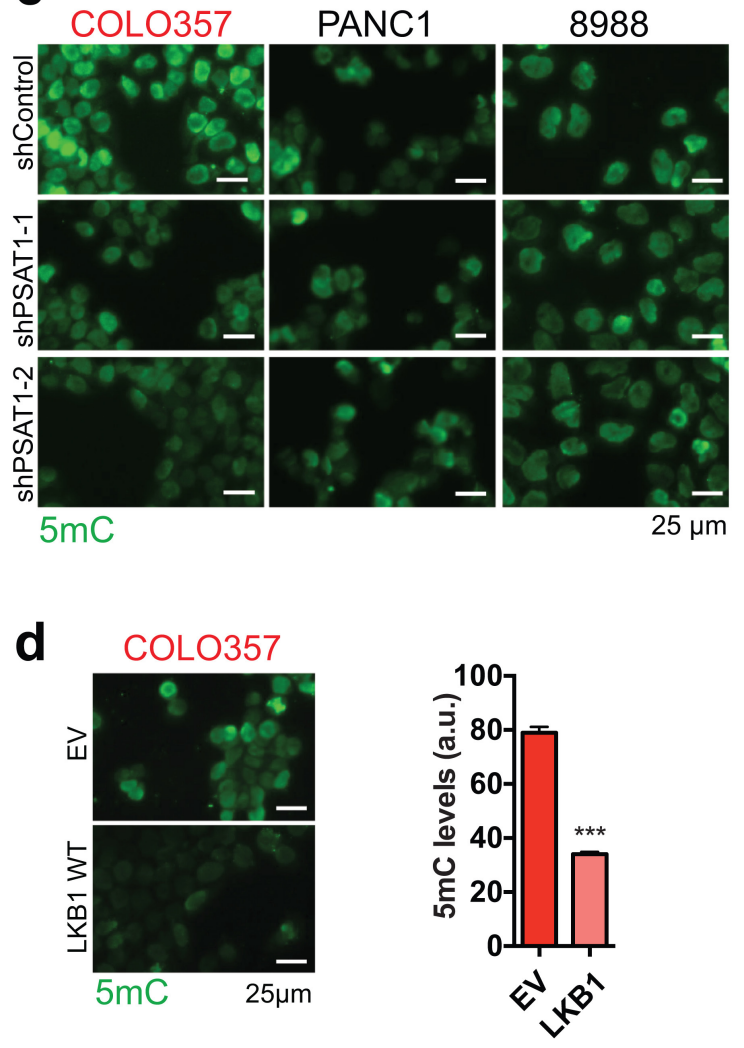

e

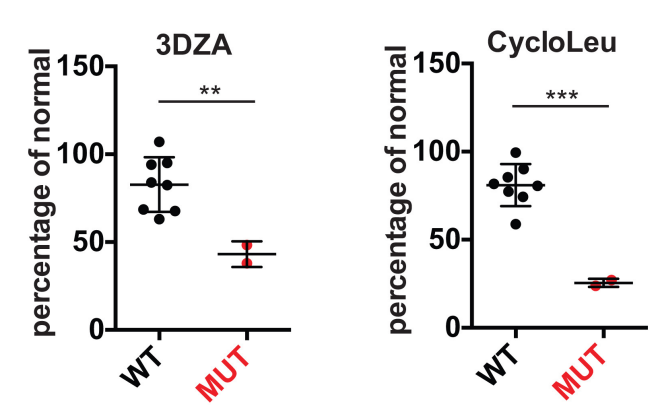

COLO357

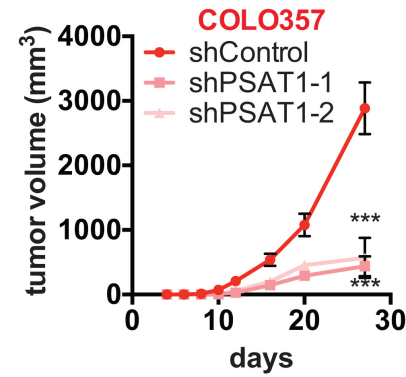

\section{$\mathrm{f}$}
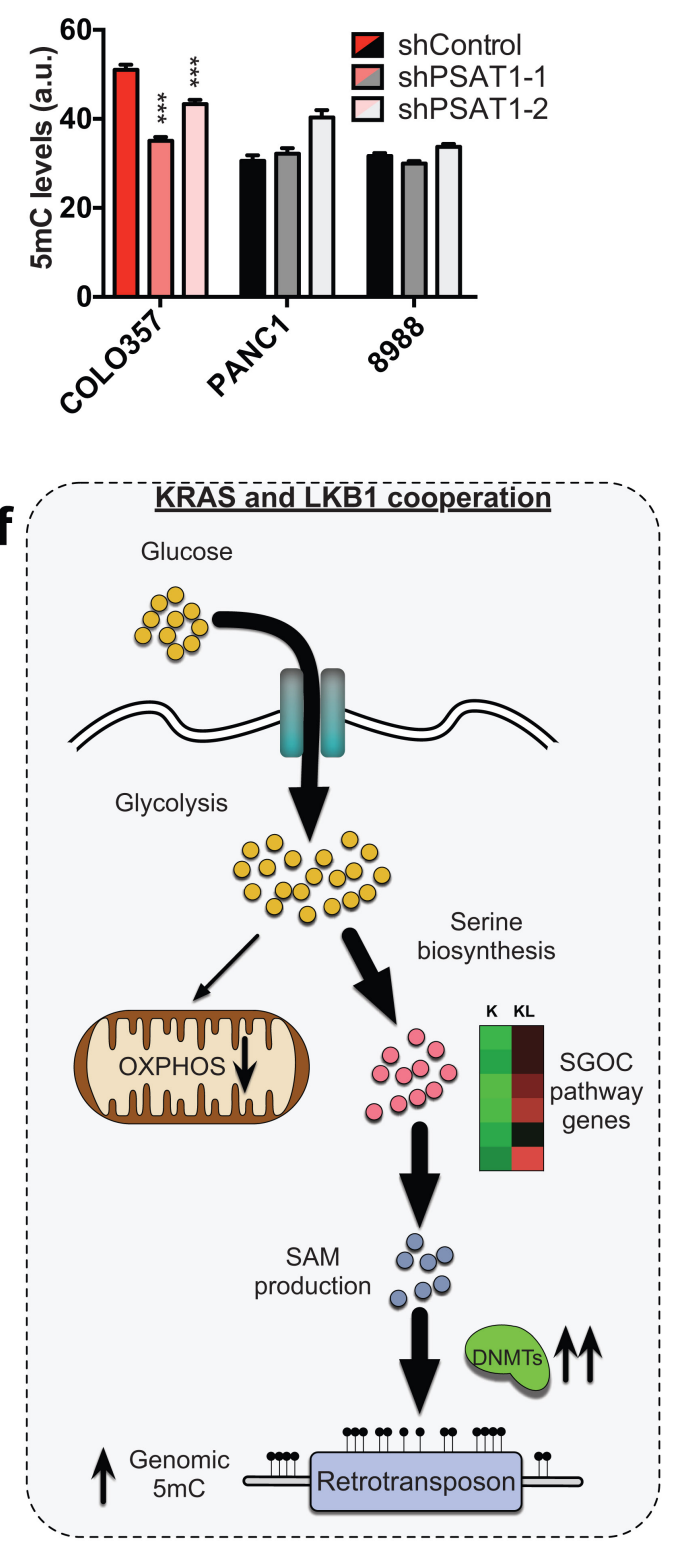

Extended Data Figure 10 | See next page for caption. 


\section{RESEARCH ARTICLE}

Extended Data Figure 10 | Vulnerabilities of human LKB1 mutant pancreatic cancer cell lines. a, Three-day growth of LKB1 wild-type (black) or LKB1 mutant (red) human pancreatic cancer cells. Data are expressed as relative to shControl-transduced cells, which is arbitrarily set to $100(n=3)$. $\mathbf{b}$, Quantification of tumour volume of subcutaneous tumours derived from implantation of the indicated cells transduced with shControl or two shRNAs against PSAT1 $(n=4)$. Error bars show s.e.m. For source data on tumour volume, see Supplementary Data Table 4.

c, Immunofluorescence staining and quantification of $5 \mathrm{mC}$ in COLO357, PANC1 and PATU-8988T cells transduced with shControl or two shRNAs against PSAT1 (607-760 cells for COLO357, 305-342 cells for PANC1 and 623-889 cells for PATU-8988T). Scale bar, $25 \mu \mathrm{m}$. Error bars show s.e.m.
Data are expressed as fluorescence per nucleus. d, Detection of $5 \mathrm{mC}$ by immunofluorescence in COLO357 (LKB1-deficient) cells transduced with vector or wild-type LKB1. Quantification is presented as fluorescence per nucleus (438-618 cells). Scale bar, $25 \mu \mathrm{m}$. Error bars show s.e.m. e, Three-day growth of LKB1 wild-type (black) or LKB1 mutant (red) human pancreatic cancer cells treated with $10 \mu \mathrm{M} 3$-deazaadenosine (3DZA) (top) or $2 \mathrm{mM}$ cycloleucine (CycloLeu) (bottom). f, Metabolic and epigenetic changes promoting transformation upon deletion of the tumour suppressor LKB1. Data pooled from (c, d) or representative of (a) two experiments. Error bars show s.d. unless otherwise stated; $* P<0.05$, $* * P<0.01, * * * P<0.001$. 